
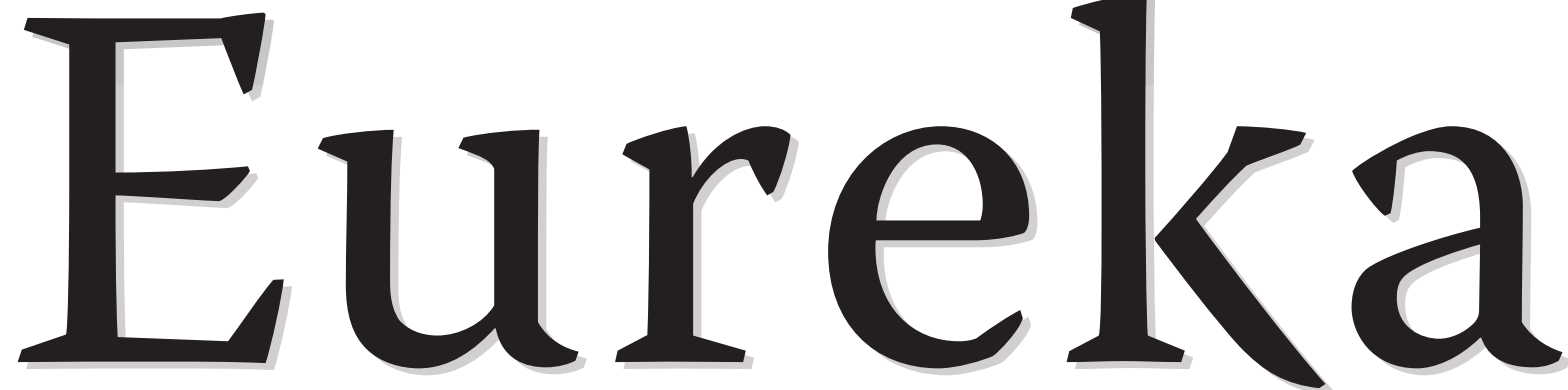

University of Alberta Science Undergraduate Research Journal http://www.eurekajournal.com

Volume 3, Number 1

February 2012

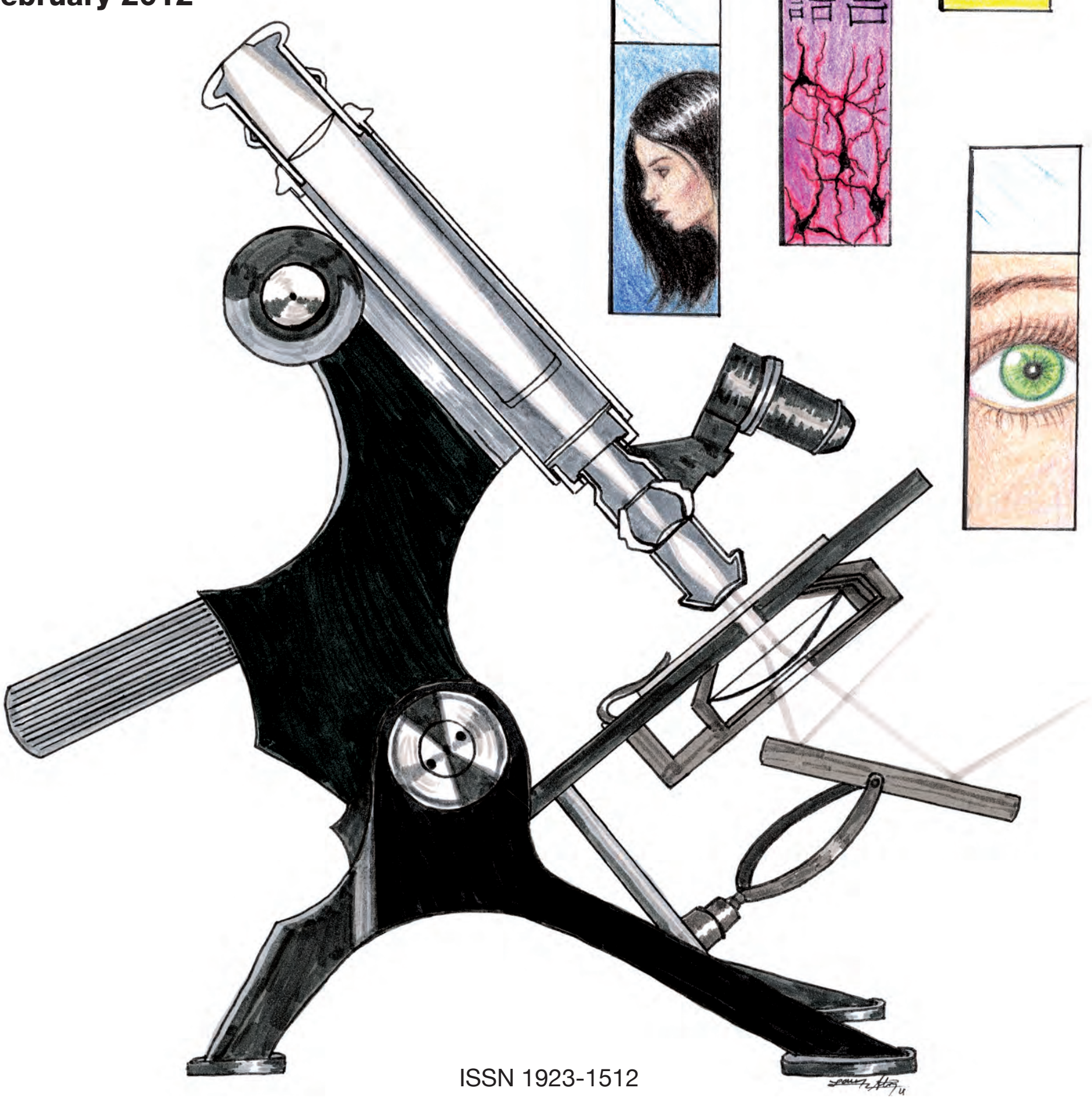




\section{EDITOR'S NOTE}

DARE TO DREAM .1

\section{INTERVIEWS}

GETTING INVOLVED IN RESEARCH: A GUIDE FOR INSPIRED UNDERGRADUATES. 2

Christopher R. Madan \& Yvonne Y. Chen

EUREKA: UNDERGRADUATES CAN PUBLISH TOO! 5

Christopher R. Madan

\section{PUBLISHED ABSTRACTS}

DEATH AND HAIRLESS CREATURES? ELUCIDATING EXISTENTIAL FACTORS IN THE EVOLUTION OF HUMAN BODY HAIR

Jordan L. Clemens, Jeff Schimel, \& David Webber

METABOLIC ENGINEERED NEUROSPORA CRASSA IS A PROMISING SOURCE FOR BIODIESEL PRODUCTION: A TEAM ALBERTA iGEM PROJECT

Kayla Baretta, Adam Foster, Yuan Guo, Murray Pelech, Raymond Odsen, Michael Simpson, Helena Zakrzewski, Douglas

Ridgway, \& Michael Ellison

THE PROPORTION OF EXCEPTION WORDS AND REGULAR WORDS IN A READING LIST INFLUENCES READING STRATEGIES IN BEHAVIOURAL AND fMRI DATA.

\section{ARTICLES}

INFRARED NON-INVASIVE SUB-WAVELENGTH MICROSCOPY WITH METAMATERIALS

Ward D. Newman

METAMATERIAL ENHANCED FLUORESCENCE DETECTION.

\section{REVIEWS}

GENDER-SPECIFIC TREATMENT FOR FEMALE YOUNG OFFENDERS 


\section{ABOUT THE FRONT COVER}

Artwork has always been a very therapeutic way of expressing my daily experiences. I enjoy using many media such as paint, pastel, pencil, and clay. Since I was a child I loved to take scraps of cardboard or wood and turn them into paintings or artistic pieces. Any form of artistic expression let me take time for myself, while producing a satisfying end product. I was always fascinated by the human body and I enjoy telling stories in my artwork through human figures. During my BSc undergraduate studies specializing in Psychology, I became very interested in turning course concepts into artwork or humorous comic strips. I figured that if I could express an academic concept in a few panels of a comic, then maybe it would create awareness of specific topics in general science and psychology. My passion for artwork and seeing the comical side to interesting concepts fuelled my desire to create a comic strip in The Gateway, the University of Alberta's student newspaper. Since 2007, my comic strip ID \& EGO ${ }^{\odot}$ has made references to topics I have come across throughout my undergraduate years such as: psychology, biological science, statistics, agriculture, political issues and popular culture.

Now as an MSc student in Neuroscience I still try to take the time to creatively express myself, whether it is a comic strip, painting, or a card for a friend. I truly believe the key to success is to keep a balanced life, especially in graduate school. Therefore not only is artwork enjoyable, but it helps manage stress and the end result is creating something beautiful, funny, or sometimes just plain odd.

The front cover image was inspired by the topics of the research papers submitted to this volume of Eureka.

\section{Lauren Alston}

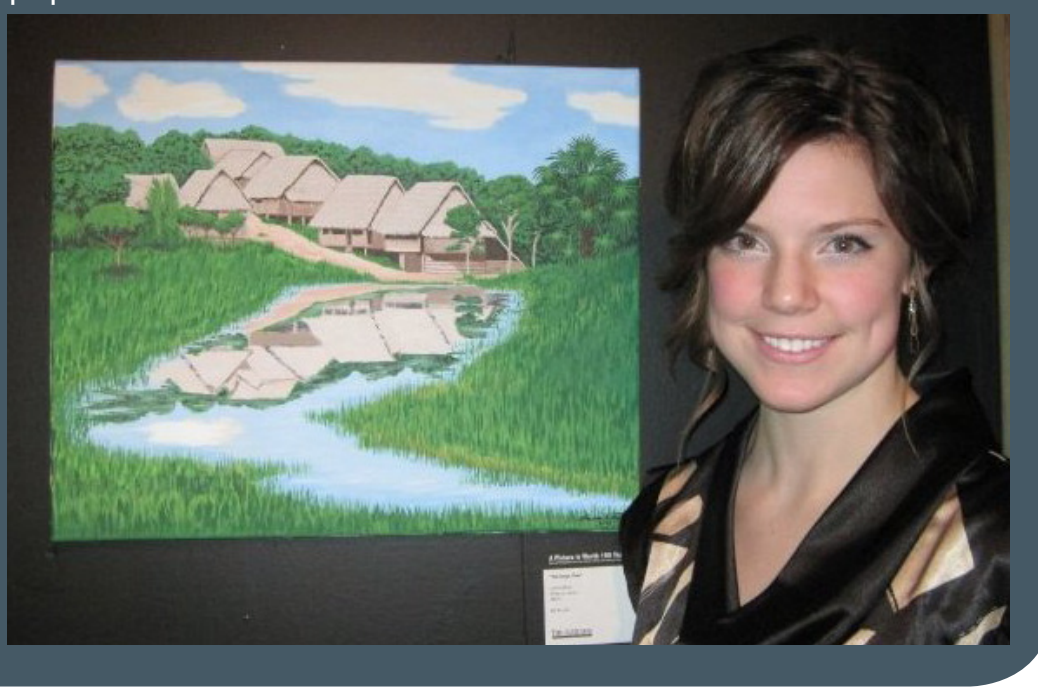

\section{ABOUT THE BACK COVER}

I grew up on an acreage situated east of Red Deer, Alberta. Growing up, I frequently found myself trying to catch things in the creek which ran through our property: wood frogs, water boatman, you name it! I do think that these times had some effect on how I directed my studies at university, but I never thought I would be conducting a wolf scat analysis for my summer NSERC research project.

Part of the project entailed creating a hair identification key to differentiate between adult and juvenile ungulates (specifically mule deer, white-tailed deer, elk, moose and bighorn sheep) seen in summer wolf diets. At the time, to my knowledge there was no mammalian hair key was able to sufficiently differentiate between juvenile and adults of the major species in the North American Wolf's diet. Presented on the back cover are only a few of the many pictures which I took under various powers of magnification to complete the hair identification key. To tell the difference between ungulate species, one mostly uses the pattern of the outside cuticle scales of the hair. This can be quite difficult and take lots of time and practice to be proficient. So, try our little hair identification quiz to see if you have what it takes.

\section{Patrick Jones}

See page 34 for answer key.

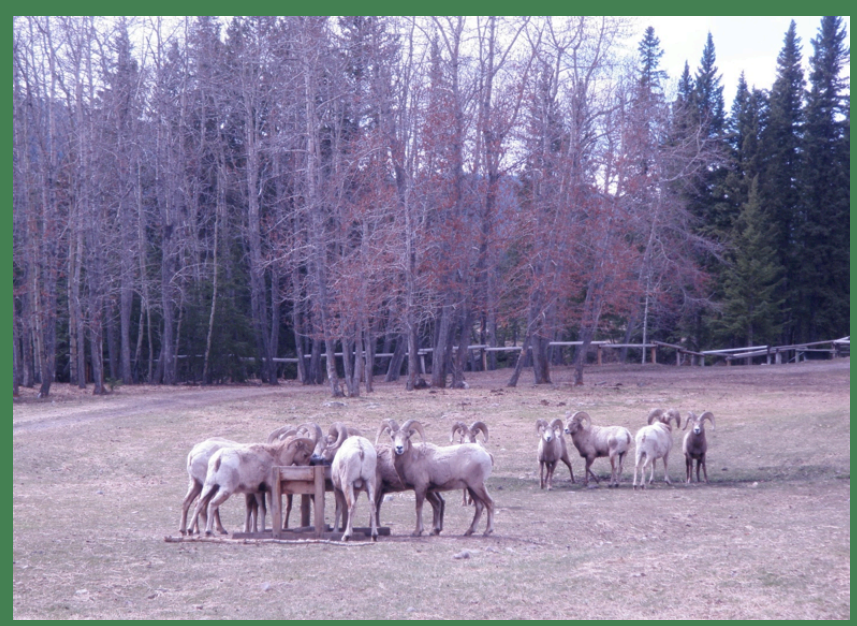




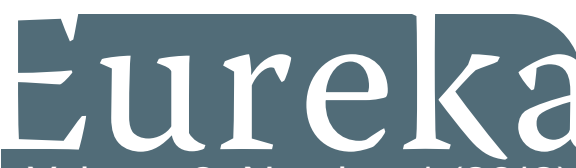

Volume 3, Number 1 (2012)

Eureka is a student-founded

and student-run initiative whose

mission is to promote the world

class undergraduate research

done in the faculty of science.

This journal offers undergraduate

scientists the unique opportunity

to share their discoveries with the

scientific world, while learning

the peer review process. Most

undergraduate students do not

get to experience the publication

process as part of their scientific

education. Eureka is an educational

institution, with a diverse team

of reviewers from many scientific

backgrounds. Through clear,

effective scientific writing students

will be able to work together with

faculty members to promote

University of Alberta science.

E-mail: eureka@ualberta.ca

Website: www.eurekajournal.com

Editor-in-Chief

Yvonne Chen

Christopher Madan

Managing Editor

Patrick Jones

Editorial Board

Yvonne Chen, Carmen Chu,

Patrick Jones, Leiah Luoma,

Christopher Madan,

Braden Teitge, Helen Zakrzewski

Layout Editors

Selena Wang

Yvonne Chen

Founders

Braden Teitge

Christopher Madan

Patrick Jones

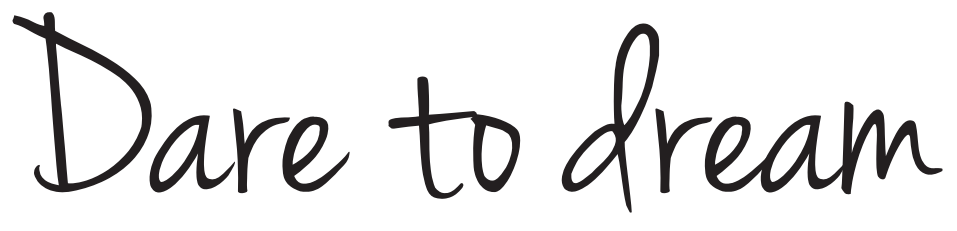

Before I came to University, I had all kinds of dreams as to what university life would be like. I imagined walking down the hall where a lab next door would be making great scientific discoveries.

When I first came to university, I was really excited. This is it! This is the place where all those wonderful research studies are happening. I suppose I was quite naïve at the time. University, especially undergraduate studies, was really not what I was expecting. I was lost in the busy hallways and large lecture halls. I then thought that would be the extent of

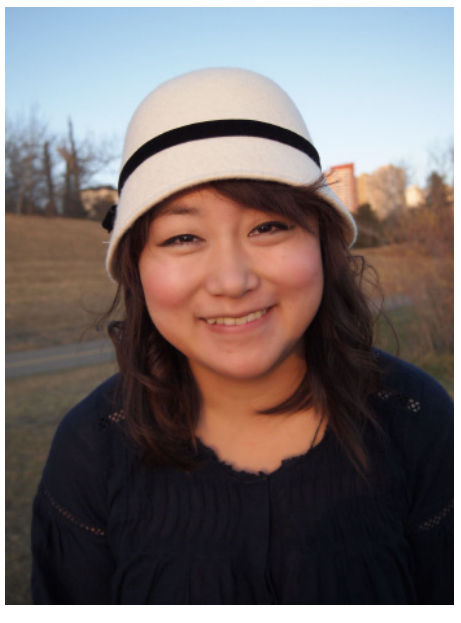
my university experience: reading about interesting experiments and exciting research in textbooks; until I stumbled upon undergraduate research. This was exactly what I was hoping for in university life! Here you are able to work on something unique, under the mentorship of an expert in the field, towards something about the world around us that is not yet known.

I was really happy when I realized that undergraduate students have the opportunity to be involved in research, and I was very glad to be apart of that. Now our university is more strongly encouraging students to become engaged in their studies by creating research-orientated courses where students can be involved in the research first-hand as an undergraduate. Earlier this year, the Student Union and the newly launched Undergraduate Research Initiative (URI) jointly organized a symposium for students to present their research. Several undergraduate journals at the University of Alberta, including Eureka, are also helping students to showcase their research to the university as a whole. Many undergraduate students are currently involved in undergraduate research, are becoming heavily involved in laboratories are undertaking interesting research, ranging from neuroscience to clinical psychology, and from electrical engineering to genetics. Here I would like to share some of their results with you in this issue.

University years are some of the most wonderful years in your life. It is a time we believe that everything is possible; dream big, aim high.

Yvonne Chen

Eureka Editor-in-Chief 


\section{GETTING INVOLVED IN RESEARCH: A GUIDE FOR INSPIRED UNDERGRADUATES}

Eureka

Volume 3, Number 1 (2012)

\section{Christopher R. Madan' \& Yvonne Y. Chen²}

1. Department of Psychology, 2. Centre for Neuroscience, University of Alberta

Most undergraduates know that the professors that teach their classes are often also researchers. But what exactly do they research? More importantly, what is research? Most departments in the Faculty of Science have research courses, not just at the 400-level, but sometimes at the 200- and 300-levels as well. Now, you're probably wondering what do students do in these classes, in research, and how can you get involved? I'm glad you asked...

\section{How can undergraduates contribute to research?}

Chris: There are many ways that you, as an undergraduate, can get involved in research. The two main ways are to take a research course or to volunteer.

Research courses. If you think you might be interested in going into research as a career, you shouldn't wait until graduate school to find out if research really is right for you. Most department in the Faculty of Science offer "individual studies courses" to undergraduates. In these courses you work one-on-one with a professor or graduate student on a particular project of theirs and learn the background for that research project, as well as how to actually *do* research. If you work with them for a term or two, you may even come up with your own research ideas and try and see if your professor would be interested in doing the study with you.

Volunteering. If you are thinking of doing a research course, but it's already into the term... that doesn't mean you have to wait until the next academic term to get involved. Most professors would be glad to take you on as a volunteer, and give you a chance to see how research is done before you completely dive in.

Roles and responsibilities. If you're either in a research course, or just a volunteer, usually you'd be expected to attend the weekly lab meetings organized by the professor who is supervising you. Usually these are meetings with the rest of the professor's research group and involve discussing a relevant research article, talking about the research project you are currently working on, and/or giving a short update on the research you have done in the last

\section{About the Authors}

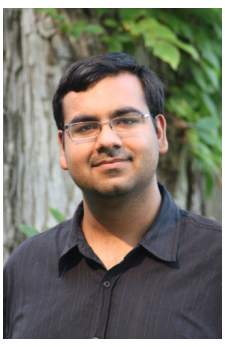

Christopher Madan first became involved in research in the second-year of his undergraduate career and is now a PhD candidate in the Department of Psychology. His research interests focus primarily on how memory is enhanced for rewarding and emotional events, and in particular how reward and emotion can influence the learning of associations. He is also interested in several other topics, including decision-making, mental imagery, and visual perception. He can be reached at cmadan@ualberta.ca.

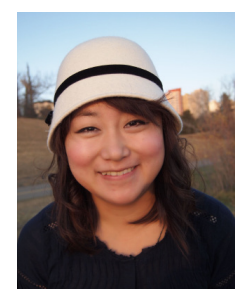

week. You are also expected to meet with the professor regularly (especially if you're doing a research course), as well as reading papers suggested by the professor. Usually some kind of data collection and analysis work is also required, but the methods used will vary greatly across the sub-fields that exist within science.

\section{Finding your passion}

\section{Yvonne: Choosing a field.}

On average, university undergraduate students in North America change their majors 3 times during the span of post secondary education. Half of the students actually do not finish their undergraduate degrees in 4 years. If you are not sure what you should do in your life, you are not alone!

If you were to ask me in my first year of university, "where do you see yourself in four years?", I can tell you without a doubt, my answer would not have been research. I came to university to study art and design. Design is such a subjective discipline. In order to create and design something truly inspirational, understanding human behaviour could be the key. While working on my designing crafts, I stumbled upon a psychology class. I liked the subject, but I wasn't particularly passionate about it, at least not until I found cognitive neuropsychology. It was the winter of my 3rd year in university, and I fell in love with every aspect of the course. This class was unique in the that I teamed up with my classmates to design and carry out a research experiment. I was excited to see the progress of the experiment and was curious to find out the results. The class changed my view of research. At the end of the semester, I approached the professor who taught the class, and he took me into his lab. I took 4 independent classes with him, and started my journey of research.

The reason of telling you about my story is hoping to demonstrate to you that you could also benefit from undergraduate research as well. Involved in undergraduate research is not as difficult as you may have imagined.

\section{Find your interest, find you passion.}

Learn from your peers. I am sure you make friends in your classes. The reason that you are taking same classes (besides filling up your prerequisites) is that you might be interested in the same subjects. Talk to them, find out what they do for classes and other school activities. You might stumble upon something might interests you.

Trying things out. Using a quote from a movie: "Life is like a box of chocolates. You never know what you're 
gonna get". How would you know what kind of chocolate is your favorite without trying any? Go out there and try all the chocolate if you can, and then you might know you like milky one more than the dark one or something else. Then choose the one you like the best! If none of them is your pick, don't stop! Keep looking till you find the one you love! It is the same to career choices or school major choices. First year students should take all those interesting courses to open their eyes, and then you can choose to specialize in one. Sometimes, it might take few tries before you might something that truly interests you.

\section{Your professors are researchers too!}

Yvonne: It can be a very scary thing to approach someone in the position of the research. Especially the only connection that you might have to the person would be the class you took, where you might be sitting at the back row, looking over almost 200 students and listening to the professor lecture over the speaker. Approach someone in the research might be easier than you might imagine.

It is very important before you approach anyone, do research on them: that might includes their research interest, recent publications, and current research projects. One hand, you can find a good match with your interests and the right professor; on the other hand, the knowledge you have about the professor would also give the professor a good first impression.

Find a good match. If you have previously taken a course with a professor, you might already have some ideas where their research interests lay. In some cases, you might find that their research interest might not be a good fit to yours. You can try to talk them and ask them for referral to other professors that might match your interests. Department websites would be a good place to begin your search. Each department website has a section for current professors, along with their research profiles. By reading them over should give you some ideas which professor you would like to approach. Things to keep in mind is that not everyone would have exact same interests as yours, the best way is to find someone that have interest that close to yours and might be able to provide the means to the kinds of research you want to do. Some department hosts "meet the prof night". It is a mixer between students and professors where you can meet the professors from that field and find out more about their research in a less format setting.

Get help. This year, University of Alberta launched Undergraduate Research Initiative (URI). It helps to facilitate undergraduates who want to get involved in research. They host free seminars, online database of undergraduate research opportunities.

Chris: Most university professors don't just teach undergraduates classes. In fact, this is very little of what they do! If you are interested in research, and have some idea of what field you want to go into, look up what kind of research your professors do. Most students usually don't have a good idea of what kind of research they are really interested in, but you probably can narrow it down a bit: Do you like chemistry or psychology? Computer science or geology? Physics or genetics? As long as you can choose a general field, you can start figuring out what kind of research you want to do. From this point, go to the department's website and look up the faculty members and read the 1-2 line descriptions of their research that are posted there. A great place to start would be the professors of the classes you found most interesting already! There's no need to try and narrow down your research interests just yet. Take advantage of the fact that you don't have a very specific research interest yet and meet with a few professors and see what (and who) inspires you!

\section{Approaching a professor about working in their lab} Chris: Okay, so you read a but about a professor and think they might be interesting to work with. Now what?

There's no reason to be worried or scared... the professor you want to meet was in your position too at one point too. If you happen to be in a class of this particular professor, one option would be to go to their office hours (preferably not near exam time) and tell them that you think their research is interesting and talk to them about what they do. Office hours not near an exam are usually not particularly busy, so you should be able to talk with the professor a bit to give you a better idea of how you interact one-on-one, rather than just watching the professor teach a class.

If you're not in a class of the professor, or are more serious, email the professor and ask if you can meet with them about working in their lab. At this time be prepared to tell the professor which aspects of their research you find particularly interesting, but also be prepared to talk about what you hope to gain by working with them, and consider bringing a copy of your transcript (an unofficial copy is fine).

Yvonne: When it comes to approaching the professor. If you have never meet this person, "email - in person visit email" approach would be a best.

First email. Writing this email is like writing a cover letter for a job. There are few elements you need to include. 1) Where did you get hold of their email addresses. Maybe from a course you took, maybe from the department website, or someone refereed to you. 2) Some information about you. That could include your name, your year, your interests, your intension of approaching this professor and so on. You may want to keep this concise. 3) Set a time to meet. Suggest that you could like to meet in person to discuss this further in person. You could also include a resume/CV in your email, so they get a better idea about you.

In person visit. Be there on time, be there prepared. We talked the importance of researching the professor before hand to find a good match. The research you have done would prepare you for this meeting. You want to learn more about this professor and his/her research, and you might want to show that you can be a good fit for the lab. For example, you know some projects this professor has been working on. You can ask about a particular project, its progress and show your interests.

Follow up email. Hopefully, you had a good meeting with the professor. First, thank the professor for the 
opportunities. Then, if you two have agreed that you are going to try out few things in his/her lab, you can express your excitement of joining the lab. If you find out this might not be a good fit for you, and professor has suggested other contacts, you can thank the professor for those contacts, and keep them in loop as to if any of those contacts work out for you. If you did end up with one of the contacts this professor suggested. Don't forget to email and thank again. This will help you build some professional network. If there is not favorable outcome from this meeting, still thank the professor for this meeting.

\section{Welcome to the wonderful world of research!}

We genuinely hope our suggestions on how to get involved with research helped you. And, if everything works out, please send us an email at eureka@ualberta.ca and tell us your story, or just give us some feedback. We wrote this article for you, the undergraduate who is interested in research, and would love to hear how things went for you!

\section{Discover Careers in Science} CAPS offers:

- Job Postings

- Career advice and information

- Resume critiques and mock intervews

- Networking events with employers

- Job Shadow Opportunties

- Science Careers Lunch Series (Winter 2012)

- Funding for leadership and professional development

- Advice on applying to medical school and graduate studies

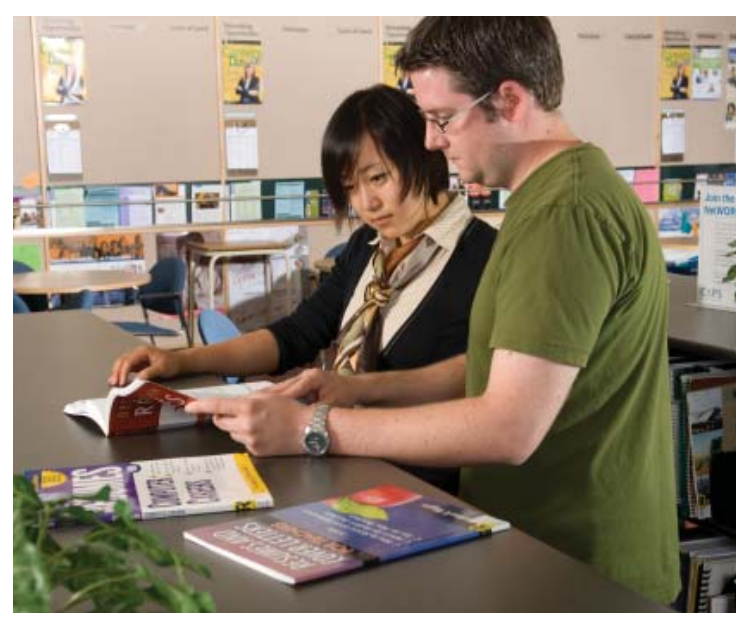

www.caps.ualberta.ca

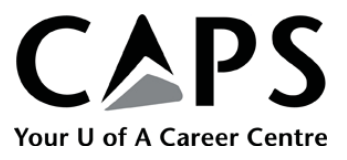


EUREKA:

UNDERGRADUATES CAN PUBLISH TOO!
Eureka

Volume 3, Number 1 (2012)

Christopher R. Madan

Department of Psychology, University of Alberta

Here you are, with a copy of Eureka in your hands. Between our covers, you can see the research of your fellow undergraduates. But, I am sure you are still left with some questions: What is the purpose of Eureka? How did Eureka get started? And most importantly: How can I submit my work to Eureka? Well, here are your answers.

\section{What is Eureka? How did Eureka get started?}

Eureka is a student-founded and student-run undergraduate science journal at the University of Alberta. It is the result of a vision that three undergraduate students had a few years ago, to serve as a platform to showcase the world-class research being done at the University of Alberta. Through Eureka, senior undergraduates can publish their work and experience the peer-review process firsthand, while more junior undergraduates can see the work of the peers and gain a better understanding of what an undergraduate can do in a research setting. Eureka began accumulating submissions in September of 2009, and now published three issues (including the one in your hands right now). The three undergraduate students I mentioned earlier are still involved in Eureka: Braden Teitge, Patrick Jones, and myself - Christopher Madan.

All three of us have graduated, but are still at the University of Alberta: Braden Teitge is now a MD student. Patrick Jones is currently pursuing a BSc in Pharmacy. I am currently working on a PhD in Psychology, with a focus on the cognitive neuroscience of memory.

\section{Why should I submit my work to Eureka?}

So you have already conducted research, either through a 200- or 400-level independent studies course, an internship, or even through a literature review in a senior class. Why should you submit your work to Eureka? By having your work published in Eureka, you can gain some experience with the peer-review process. While many professors point out the importance of the peerreview process in their courses, students rarely get the opportunity to experience it firsthand. Papers submitted to Eureka are reviewed by two reviewers (often graduate students, but sometimes faculty members as well), and authors are provided with feedback to revise their work as is done with 'full scale' journals. Additionally, you can list the publication on your CV when you apply to graduate school, and can include a copy of your paper with your submission. Often undergraduates have not published any research by the time graduate school admission deadlines come around, so having published your work in any form will greatly help your application.

\section{What kind of submissions does Eureka accept?}

We accept three types of submissions: research articles, published abstracts, and reviews.

Research articles are what you usually think of when you talk about research papers. These are the primary results published by researchers, where an experiment was conducted and data was analyzed and interpreted. These types of papers are often the result of an 400-level independent studies course or summer research internship.
Published abstracts are based on the same work as a research article. However, some 'full scale' journals may have an issue if your work was first published with us as a research article prior to being submitted to them. To address this issue, we allow you to publish just an abstract of your work with us. The abstract is like 1-3 pages in length, with less detail included on the exact experimental method and analyses, and instead focusing on the study's initial motivation and an interpretation of the results. These abstracts are similar to what is often submitted to for a conference's proceedings.

Reviews are often the term papers you write for a 400 -level course. These involve doing a thorough literature review of a given topic, and combining the results of these studies to point a general finding across a large number of studies. However, literature reviews do not need to have been done in a senior-level course, as demonstrated by a recent Science 100 student (see Polet, 2011, Eureka 2, p. 43-46).

In all of these cases, please see works that have been previously published in Eureka to get a better idea of what a completed article looks like.

\section{When can I submit my work to Eureka?}

Eureka has several "call for submissions" per year. However, we do accept submissions at any time. As previously mentioned, submissions will be reviewed and the author will need to make revisios prior to the submission being accepted. Thus, it is prudent to submit your work early so that it will not need to be delayed until the following issue.

You can submit your work either through our website (http://www.eurekajournal.com) or by emailing your submission to us at eureka@ualberta.ca. Additionally, please email us if you have any questions about submitting your work to Eureka.

\section{How can I become involved with Eureka?}

Obviously, the best way is to submit your work to us! However, if you want to become even more involved, you could become an editor. Editors should be in at least their third-year of undergraduate studies, and have a strong background in their field. If you are interested, please email us and we will discuss the roles and responsibilities with you further.

If you do not yet have a strong background in a given field, but still want to become involved with Eureka, we do have other positions as well. To make Eureka what it is, we also need the help of layout editors, copy editors, as well as general volunteers. Layout editors convert the 'boring' looking double-spaced manuscripts we receive into the professionally typeset journal you are reading right now. Copy editors proof the text for grammatical and typographical errors. General volunteers help us fundraise, advocates for Eureka, and advertise our call for submissions. If you are interested in any of these positions, do not hesitate to email us and we can discuss the positions with you further. 

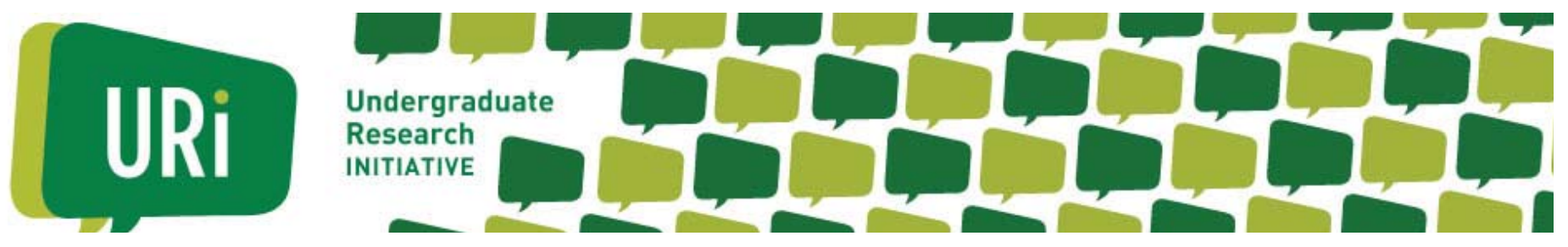

\section{Ever wonder why the sky is blue, what a black hole is, or why music moves us?}

Answer your questions with the Undergraduate Research Initiative (URI)! The URI wants to help you learn how and why to get involved in research and creative works at the $U$ of $A$. As an undergraduate student researcher, you can:

- Make your degree stand out

- Develop connections with faculty

- Investigate topics you're passionate about

Here are some of the URI services available to you:

- Online resources and information

- Inventory of Undergraduate Research Opportunities

- $\quad$ Undergraduate research funding

- $\quad$ Panels and presentations

- $\quad$ Brokering and faculty advising

- $\quad$ E-newsletter of opportunities and events

- $\quad$ Field trips

- $\quad$ Physical meeting space

\section{www.uri.ualberta.ca}




\section{DEATH AND HAIRLESS CREATURES? ELUCIDATING EXISTENTIAL FACTORS IN THE EVOLUTION OF HUMAN BODY HAIR}

Eureka

Volume 3, Number 1 (2012)

Jordan L. Clemens, Jeff Schimel, \& David Webber

Department of Psychology, University of Alberta, Edmonton, Alberta

\section{Introduction}

Human hairlessness is something of an anomaly in the animal kingdom. Why did human beings lose their body hair? Why are we the only primates with nearly naked skin? The evolutionary theories that have been advanced so far run the gamut, from parasitic evasion to a brief phase as an aquatic animal (for a review of the theories, see Wade, 2003; Jablonski, 2010). However, despite all of the theorizing done so far, the exact evolutionary pressures that led to the loss of body hair still remains a mystery. The purpose of the following research was to test a psychological hypothesis for why humans may have lost their body hair, which is derived from Terror Management Theory (TMT; Greenberg, Pyszczynski, \& Solomon, 1986) theorizing and research.

\section{Terror Management Theory}

Inspired by the works of cultural anthropologist Ernest Becker (e.g., The Birth and Death of Meaning, 1971; The Denial of Death, 1973; Escape from Evil, 1975), TMT was developed as a social psychological, empirical extension of Becker's psychoanalytic and existential ideas. Becker, as well as TMT, starts with two broad assumptions: (1) humans are like all other animals in that we have a biological propensity for continued existence, and that (2) we are unlike other animals in that our advanced cognitive architecture leaves us uniquely aware of the inevitability of death. That is, human beings share a great deal with our animal brethren, but the one thing that really marks us off as unique is our extreme capacity for self-reflective thought; the ability to be aware of ourselves as beings existing over time, with both a beginning (birth) and an end (death). This paradox of constantly being driven towards existence, but being aware that life is finite, has the potential for debilitating terror: a terror that has to be "managed".

To manage this terror, Becker theorized that we create and maintain "systems" of meaning to protect us from our existential anxiety. These systems are culture, which Becker broadly defines as a shared, symbolic, and mutually constituted (enforced) conception of reality. Stated with brevity: culture provides us with meaning and a sense of self-esteem in the face of death (see Pyszczynski, Greenberg, Solomon, Arndt, \& Schimel, 2004). Furthermore, participation in a system that is larger than one's self, a system that seems to be omnipotent and eternal, allows the individual to repress their death anxiety, and attain immortality literally (i.e., a promise of a soul or immortality) or symbolically (i.e., living on in the mind of the culture writing the Great Novel, being someone of prominence, being a hero, etc.). Thus, from a TMT analysis, we can see the importance of culture and self-esteem in the face of our existential condition.

\section{Terror Management Research}

Drawing from the aforementioned theories, social psychologists have conducted more than 300 empirical studies to date testing TMT hypotheses in several different countries, and in a multitude of different settings (see Burke et al., 2010, for a comprehensive review). One of the most fundamental of these TMT hypotheses is the mortality salience hypothesis (hereafter, MS), which simply states: if culture serves a death denying function, then individuals who are experimentally induced to think of their death should have a greater need to affirm and protect their worldview (worldview defense), and derogate or have disdain for people with a differing worldview. That is, if we are existentially and affectively invested in our deathdenying worldviews, then we are inevitably disposed to suspend and defend them dogmatically. These results have been obtained in a multitude of studies cross culturally (Greenberg et al., 1997).

Creatureliness/Animality. Building on the general theory laid out by TMT, several researchers have pushed the idea further, and have begun to look at how our concerns about death factor into our appraisals of the body and human sexuality (Goldenberg, Pyszczynski, Greenberg, \& Solomon, 2000), human beings as unique from animals (Goldenberg et al., 2001), disgust reactions (Cox, Goldenberg, Pyszczynski, \& Wiese, 2007) and even romantic partner selection (Kosloff, Greenberg, Sullivan, \& Wiese, 2010). The general consensus from these studies is that the body is existentially problematic for humans, since it is both the source and place of everything we experience, and the end of all that experience; the fact that we are bodies means that we are always beings towards-death.

Study Hypothesis. Once humans were fully self-aware, physical features such as body hair may have been a potent reminder of human creatureliness (and therefore human vulnerability to death). If this is true, body hair as a physical trait may have been inhibited through sexual selection. Given that the act of sex is also a reminder of human creatureliness (Goldenberg et al., 1999; Landau et al., 2006), it is entirely possible that sexual selection could have led to a reduction in body hair over a relatively short period of time. If this analysis is correct, when people are briefly reminded of their own mortality, they should show stronger sexual attraction to a potential partner with less (vs. more) body hair, in order to distance themselves from their creatureliness and mortality. The research described below was designed to test this hypothesis. 


\section{Study (Methods and Design)}

Overview. To test our hypothesis, we amassed a variety of pictures of university-age looking men, and manipulated them with Photoshop to look more or less creaturely via four different gradations of body hair (the first gradation being the least hairy, and the fourth being the most hairy). The participants were asked to rate the pictures on several different dimensions of attraction (note: level of attractiveness was controlled for in an earlier pilot study to find the best pictures). The main question of interest was: How sexually attractive is this person?

Participants and design. 77 undergraduate, heterosexual women in introductory psychology at the University of Alberta participated in our study. Participants were randomly assigned to a 2 (MS vs. control) X 2 (body hair level) mixed factorial design. Those in the MS condition were induced to think of their own death, whereas those in the control condition were induced to think about dental pain. Only women were included in this preliminary analysis, since women - historically, socially and biologically - have much more to invest (and therefore to lose) in mate selection than men. Thus, we reasoned they are more likely to drive the sexual selection process.

Participants were told the study was looking at various personality characteristics and their relation to interpersonal attraction. Participants then filled out a personality packet, which contained the MS vs. control (dental pain) manipulation amongst the filler personality questions. After a brief delay, they were then asked to complete the attraction task, which consisted of rating their attraction to various pictures of men with varying levels of body hair.

\section{Results and Discussion}

Consistent with our hypothesis, we found that those participants who were reminded of their death significantly preferred hairless models to hairy models, whereas those in the control condition showed no significant preference. Thus, the participants' attraction to potential mates was amenable to existential concerns. When the participants' mortality was salient, they sought to distance themselves from their own animality ("flee the body") by preferring a male model that was "humanized" by virtue of his hairlessness. Thus, it seems that we have fertile preliminary data to tentatively suggest that existential concerns may have played a role in the sexual selection of hairless (or nearly hairless) humans. If, as embodied creatures living in expansive realms of meaning and purposive action, we always seek to deny that which we are, it seems reasonable that these concerns may have plagued the burgeoning human species, and may have (in part, at least) led to the selection of certain human characteristics we see today.

In sum, our preliminary analysis and theorizing suggests that human beings are every bit as existentially motivated, as they are biologically motivated. We are always, as Becker quipped, "angels with anuses": hybrid spiritualbiological beings forever ebbing between the ethereal and mundane; tirelessly creating, expanding, and transcending nature, and yet ultimately, we are still bounded bodies born to die.

\section{References}

Becker, Ernest (1971). The birth and death of meaning (2nd ed.). New York, NY: The Free Press.

Becker, Ernest (1973). The denial of death (1 $1^{\text {st }}$ ed.). New York, NY: The Free Press.

Becker, Ernest (1975). Escape from evil (1 ${ }^{\text {st }}$ ed.). New York, NY: The Free Press.

Burke, B. L., Martens, A., \& Faucher, E. H. (2010). Two decades of terror management theory: A meta-analysis of mortality salience research. Personality and Social Psychology Review, 14(2), 155-195.

Cox, C.R., Goldenberg, J.L., Pyszczynski, T., \& Weise, D. (2007). Disgust, creatureliness and the accessibility of death-related thoughts. European Journal of Social Psychology, 37, 494-507. doi:10.1002/ejsp.370

Goldenberg, J.L., Pyszczynski, T., McCoy, S. K., Greenberg, J., \& Solomon, S. (1999). Death, sex, love, and neuroticism: Why is sex such a problem? Journal of Personality and Social Psychology, 77, 1173-1187. doi:10.1037/0022-3514.77.6.1173

Goldenberg, J.L., Pyszczynski, T., Greenberg, J., \& Solomon, S. (2000). Fleeing the body: A terror management perspective on the problem of human corporeality. Personality and Social Psychology Review, 4, 200-218. doi:10.1207/S15327957PSPR0403_1

Goldenberg, J. L., Pyszczynski, T., Solomon, S., Greenberg, J., Kluck, B, \& Cornwell, R. (2001). I am not an animal: Mortality salience, Disgust, and the denial of human creatureliness. Journal of Experimental Psychology, 130, 427-435.

Greenberg, J., Pyszczynski, T., \& Solomon, S. (1986). The causes and consequences of a need for self-esteem: a terror management theory. In R. F. Baumeister (Ed.), Public self and private self (pp.189212). New York: Springer-Verlag.

Greenberg, J., Solomon, S., \& Pyszczynksi, T. Terror management theory of self-esteem and cultural worldviews: Empirical assessments and conceptual refinements. (1997). In Zanna, Mark P [Ed]. (1997). Advances in experimental social psychology, Vol. 29. (pp. 61-139). ix. San Diego, CA, US: Academic Press; US.

Jablonski, Nina G. (2010, February). The naked truth: why humans have no fur. Scientific American, 302(2), pp. 42-49.

Kosloff, S., Greenberg, J., Sullivan, D., \& Weise, D. (2010). Of Trophies and pillars: Exploring the terror management functions of short and long term relationship partners. Personality and Social Psychology Bulletin, 36, 1037-1051.

Pyszczynksi, T., Greenberg, J., Solomon, S., Arndt, J., \& Schimel, J. (2004). Why do people need self-esteem?: A theoretical and empirical review. Psychological Bulletin, 130, 435-468. doi:10.1037/00332909.130.3.435

Rozin, P., Haidt, J., \& McCauley, C. R. Disgust. (2008). In Lewis, Michael [Ed]; Haviland-Jones, Jeannette M [Ed]; Barrett, Lisa Feldman [Ed]. (2008). Handbook of emotions. (3rd ed.). (pp. 757-776). New York, NY, US: Guilford Press.

Wade, Nicholas. (August 19, 2003). Why Humans and Their Hair Parted Ways. Retrieved from: http://www.nytimes.com/2003/08/19/science/ why-humans-and-their-fur-parted-ways.html?scp=1\&sq=why $\% 20$ humans\%20and\%20their\%20fur\%20parted\%20ways\&st=cse 
METABOLIC ENGINEERED

NEUROSPORA CRASSA IS A PROMISING

SOURCE FOR BIODIESEL PRODUCTION:

Eureka

Volume 3, Number 1 (2012) A TEAM ALBERTA IGEM PROJECT

Kayla Baretta, Adam Foster, Yuan Guo, Murray Pelech, Raymond Odsen, Michael Simpson, Helena Zakrzewski, Douglas Ridgway, \& Michael Ellison

iGEM, University of Alberta, Edmonton, Alberta

Our current transportation infrastructure is heavily reliant on traditional fossil fuel sources. Developing sustainable alternative fuels is the most important challenge to reversing this trend. In Alberta, besides the production of fossil fuels, there is an abundance of cellulosic biomass from the forestry and agricultural industries. This presents the opportunity to develop a biofuel production process utilizing waste biomass, including grass clippings and excess agricultural straw, to lower energy costs and reduce carbon emissions.

To address this challenge, we propose to metabolically engineer the filamentous fungus, Neurospora crassa, a natural cellulose metabolizer, to produce high levels of fatty acids. In turn, the fatty acids will be chemically modified to fatty acid methyl esters (FAMEs), more commonly known as biodiesel. FAMEs have been commercially tested as substitutes for traditional fossil diesel in all modern engines.

To increase the fatty acid content of Neurospora crassa, we will be attempting a simultaneous knockout of fadD1 gene and an insertion of a codon optimized TesA' gene, a thioesterase from $E$. coli by homologous recombination. The fadD1 knockout will impair fatty acid degradation through $\beta$-oxidation. Furthermore, TesA' cleaves fatty acyl chains from ACP domain of the fatty acid synthase (FAS) complex reducing the inhibition of the FAS complex. .

The chemical conversion of fatty acids to FAMEs is through acid catalyzed esterification using $\mathrm{MeOH}$ and $\mathrm{HCl}$. In this process, the collected $N$. crassa mass can be directly added to the reaction vessel without the prior extraction of the fatty acids.

Figure 1. The genetic construct used to transform N. crassa. It consists of four pieces. The 5' upstream region and 3' downstream region around the fadD1 are points for homologous recombination. The synthetic tesA' gene encodes for the thioesterase to reduce inhibition of the FAS complex. The HygB gene encodes for resistance to hygromycin B allowing for selection of transformed cells. 


\title{
THE PROPORTION OF EXCEPTION WORDS AND REGULAR WORDS IN A READING LIST INFLUENCES READING STRATEGIES IN BEHAVIOURAL AND fMRI DATA
}

\author{
Crystal Zhou \& Jacqueline Cummine \\ Department of Speech Pathology \& Audiology, University of Alberta, Edmonton
}

There has been considerable functional neuroimaging support for a dual-pathway neuroanatomical model of reading that distinguishes between a ventral whole-word or lexical stream and a dorsal sub-word or sublexical stream. The relative contribution of these two streams while participants read aloud familiar stimuli, however, still remains unclear. This study investigated the relative involvement of the dorsal and ventral streams during reading of highly familiar stimuli by manipulating the proportion of regular words (REGs; stimuli that can be correctly processed by both ventral and dorsal streams) and exception words (EXCs; stimuli that can only be correctly processed by the ventral stream). The behavioural evidence supported modulation of lexical and sublexical pathway contributions. Specifically, when $75 \%$ of the words were REGs, both lexical and sublexical information were utilized, as evidenced by the fast reaction times and increased errors for EXCs. In contrast, when $75 \%$ of the words were EXCs, participants minimized sublexical processing, as evidenced by fast reaction times and decreased errors for EXCs. Neuroanatomical evidence provided further support, such that reading a REG-predominant list induced recruitment of both ventral and dorsal stream regions, while reading an EXC-predominant list induced recruitment of the ventral stream and the additional employment of a phonological lexical check (via BA6) as response modulation. These results support parallel operation of the dorsal and ventral stream and provide evidence that the extent to which each stream contributes to reading can be modulated

Figure 1. Ventral stream associated areas in yellow and dorsal stream-associated areas in blue. 1. BA6 2. Temporoparietal junction (supramarginal gyrus, angular gyrus, posterior superior temporal gyrus). 3. Inferior occipitotemporal region. 4. Middle and inferior temporal gyri.

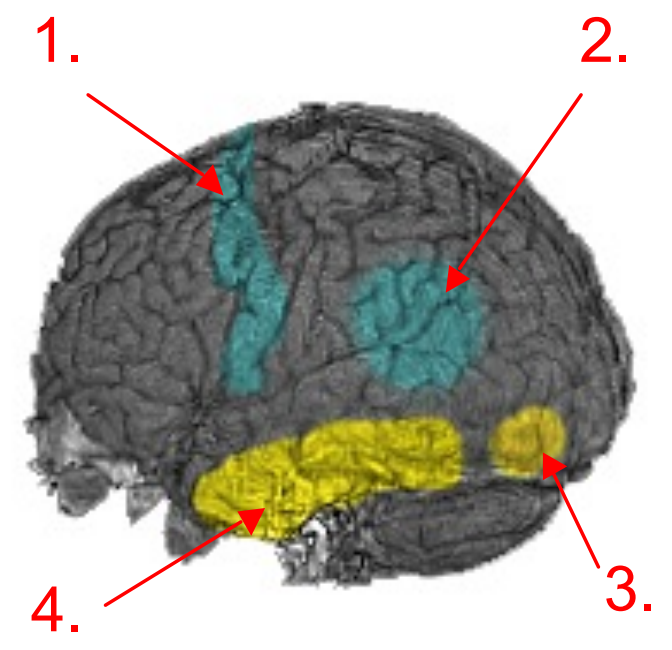




\section{INFRARED NON-INVASIVE SUB-WAVELENGTH MICROSCOPY WITH METAMATERIALS}

Ward D. Newman

Electrical and Computer Engineering, University of Alberta
Eureka

Volume 3, Number 1 (2012)

\begin{abstract}
I demonstrate that hyperbolic metamaterials may provide the solution to the long-standing problem of the fundamental diffraction limit plaguing conventional microscopy and optical imaging systems. Presented here is the formalism of the classical electrodynamics used to describe the diffraction limit and sub-wavelength imaging using hyperbolic metamaterials. Effective medium theory is then derived for planar multilayer systems and put forth as a design method for such hyperbolic metamaterials; that is, multilayer systems consisting of alternating layers of metal and dielectric have an extreme anisotropy in the bulk dielectric tensor, leading to hyperbolic spatial dispersion. I then outline the design of a hyperbolic metamaterial base device for use in sub-wavelength infrared microscopy, and present numerical simulations to demonstrate the behaviour and performance of the device. The device employs multilayers of InGaAs/ AllnAs and is capable of sub-diffraction imaging resolution in the wavelength range of $8.8-10.5 \mu \mathrm{m}$. I show that high spatial frequency waves, which normally decay in vacuum, are allowed to propagate and reach the far-field in a hyperbolic metamaterial. Using a Green's function formalism to describe optical sources, sub-wavelength imaging capabilities of hyperbolic metamaterials is shown. Finally, potential device applications using the designed metamaterial are motivated.
\end{abstract}

\section{Introduction}

Microscopy and imaging has played a monumental role in modern science, and continues to do so. Biologists and many other fields of science have employed, what is now conventional optics, since at least the seventeenth century [1]. Even now, modern nano- and micro-fabrication facilities still rely heavily on basic to advanced microscopes for imaging and characterizing a wide range of objects such as integrated electrical circuits (ICs) and micro electromechanical devices (MEMs). In addition, one of the revolutionary micro-fabrication techniques, photolithography, relies on the physical principles of classical optics [2]. Nevertheless, there is still one major hurdle to overcome with optical microscopy and related technolo- gies, namely, the diffraction limit.

The diffraction limit is a physical effect which restricts the smallest feature size that can be resolved in an image to about half the imaging wavelength, $\sim \lambda / 2$. In terms of the optical spectrum ( $400-800 \mathrm{~nm})$, this limits the smallest resolvable feature size to $\geq 200 \mathrm{~nm}$. This physical phenomena is well explained using classical electrodynamics and Fourier optics; however, current technologies which can overcome this diffraction limit or which allow sub-wavelength (optical) resolution are either destructive (such as scanning electron microscopy and transmission electron microscopy) or invasive (atomic force microscope and scanning tunnelling microscope).

The emerging field of metamaterials may provide the solution to overcoming the diffraction limit through the ability to control and manipulate fundamental lightmatter interactions. This is achieved by tailoring the bulk and macroscopic material properties by controlling the sub-wavelength structure of a material. Twentieth century advances in nanofabrication have allowed the nanosctructure of devices to be tailored at will so that the fabrication of these metamaterials and their exotic electromagnetic properties are now possible [3]. In natural materials, the bulk and effective properties of a material is governed by the local arrangement (domain) of atoms, and the atomic lattice. A metamaterial is an artificially structured material with sub-wavelength structures known as meta atoms which govern the bulk electromagnetic properties as shown in figure 1.

In particular, Hyperbolic Metamaterials (HMMs) have shown great promise to overcoming the diffraction limit $[3,4]$ by allowing evanescent (decaying) EM waves which cannot be imaged using conventional optics, to propagate and hence be detected and imaged. The exotic property in HMMs which gives rise to this phenomena is an unbounded, hyperbolic dispersion relation. In natural transparent materials, the dispersion relation is bounded and spherical or ellipsoidal in shape.

Outlined here is the physics necessary to analyze the principles of sub-wavelength imaging and overcoming the diffraction limit, understanding the theory governing $\mathrm{HMMs}$, and designing and testing the performance of a HMM for use in the infrared (IR) region of the EM spectrum. The design of a HMM based device employing multilayers of InGaAs/AllnAs is summarized along with simulations of the performance of the device. These materials were first proposed for use in HMMs by Hoffman [5], however the scope of that work was on negative refraction; therefore, the theoretical and design developed and presented here, for applications in sub-wavelength imaging, is new and state-of-the-art.

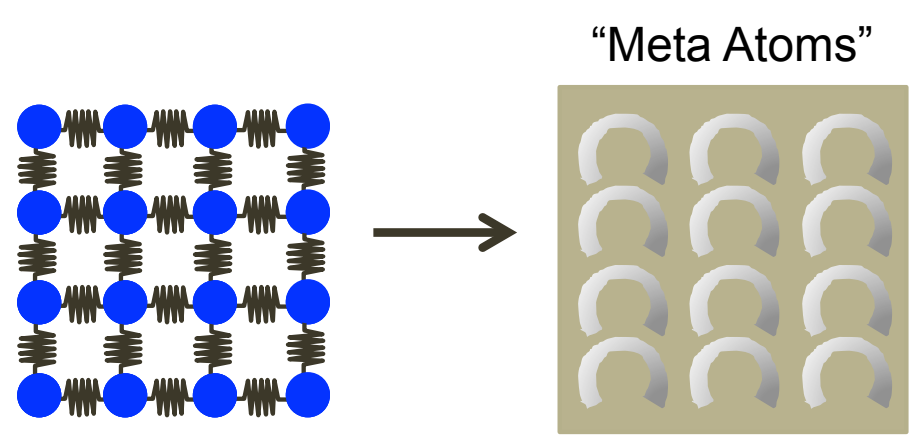

FIG. 1 In natural materials, the bulk EM properties are governed by the arrangement of atoms. In metamaterials, the bulk EM properties are governed by artificially structured "Meta atoms". 


\section{Sub-Wavelength Imaging in the IR}

Plane Wave Propagation in Anisotropic Uniaxial Media

Maxwell's equations for the time dependent electric and magnetic fields can be decoupled to yield the wave equations for EM waves, that is light in a sourceless region

$$
\left(\nabla \times \nabla \times+\frac{\overline{\mu \epsilon}}{c^{2}} \frac{\partial^{2}}{\partial t^{2}}\right)\left\{\begin{array}{l}
\vec{E} \\
\vec{B}
\end{array}\right\}=0
$$

where $\mathrm{c}$ is the speed of light, and $\bar{\mu}$ and $\bar{\epsilon}$ are the relative magnetic permeability and electric permittivity tensors respectively.

In the optical and IR region of the EM spectrum, almost all materials do not have a magnetic response so it is safe to assume $\bar{\mu}=\bar{I}$, the identity tensor. In addition, if the medium is uniaxial, then the dielectric tensor is of the form

$$
\bar{\epsilon}=\left(\begin{array}{ccc}
\epsilon_{\|} & 0 & 0 \\
0 & \epsilon_{\|} & 0 \\
0 & 0 & \epsilon_{\perp}
\end{array}\right)
$$

where the dielectric constant along the $x$-and $y$-directions is $\varepsilon_{x}$, and the dielectric constant along the $z$-direction is $\varepsilon_{z}$. This type of material has a planar symmetry in the xy-plane and the dielectric tensor has two unique components, namely $\varepsilon_{\|}$in the xy-plane and $\varepsilon_{\perp}$ in the direction normal to the $x y$-plane.

These wave equations give rise to plane wave solutions of the form

$$
\left\{\begin{array}{l}
\vec{E}(\vec{r}, t) \\
\vec{B}(\vec{r}, t)
\end{array}\right\}=\left\{\begin{array}{l}
\vec{E}_{0} \\
\vec{B}_{0}
\end{array}\right\} e^{i \vec{k} \cdot \vec{r}} e^{-i \omega t}
$$

provided that the propagation wavevector $\vec{k}$, satisfies the equation [6]

$$
\vec{k} \times\left(\vec{k} \times \vec{E}_{0}\right)=\left(\frac{\omega}{c}\right)^{2} \bar{\epsilon} \vec{E}_{0}=k_{0}^{2} \bar{\epsilon} \vec{E}_{0}
$$

This describes a wavevector surface from which the details of propagation of different types of $\vec{E}_{0}$ polarizations in the media are obtained.

From equations 3 and 4 , it then follows that for uni-axial materials described by equation 2, there are two unique types of plane waves described in equation 3: (a) Where $\vec{E}_{0}$ is parallel to the $x y$-plane and (b) where $\vec{B}_{0}$ is parallel to the $x y$-plane. These correspond to transverse electric TE polarizations (ordinary waves) and transverse magnetic polarizations TM (extraordinary) respectively. The relation between $\vec{E}_{0}$ and $\vec{B}_{0}$ for each case are easily obtained through Maxwell's Equations: $\nabla \times \vec{E}=-\frac{1}{c} \frac{\partial \vec{B}}{\partial t}$ and $\nabla \times \vec{B}=\frac{\bar{\epsilon}}{c} \frac{\partial \vec{E}}{\partial t}$. For the remainder of this discussion, only TM polarization will be treated, however, analogous arguments and derivations can performed for TE waves.

For TM polarized light, equation 4 reduces to the well known dispersion, or isofrequency relation for extraordnary waves in an uniaxial medium

$$
\frac{k_{x}^{2}+k_{y}^{2}}{\epsilon_{\perp}}+\frac{k_{z}^{2}}{\epsilon_{\|}}=k_{0}^{2}
$$

with $k_{0}=2 \pi / \lambda=\omega / c$.

\section{Diffraction Limit}

Vacuum and most natural dielectrics (glasses) are not uniaxial, but isotropic so that $\epsilon_{\|}=\epsilon_{\perp}=\epsilon>0$

Equation 5 then becomes

$$
k_{x}^{2}+k_{y}^{2}+k_{z}^{2}=\epsilon k_{0}^{2}
$$

and the wave vector surface described by equations 5 and 6 is a sphere ${ }^{1}$. In terms of imaging,we wish for an object in the $x y$-plane to be focused and imaged in the $x^{\prime} y^{\prime}$-plane, some distance $z$ from the object, figure 3. From Fourier Optics, the electric field corresponding to the object in the $x y$-plane (WLOG assume $z=0$ ) can be decomposed into plane waves (spatial frequencies) $k_{x}$ and $k_{y}$ through a Fourier transform into a Fourier (or angular) spectrum as [7]

$$
\begin{gathered}
\widehat{E}\left(k_{x}, k_{y}, z=0\right)= \\
\iint_{-\infty}^{+\infty} E(x, y, z=0) e^{i\left(k_{x} x+k_{y} y\right)} d x d y
\end{gathered}
$$

This Fourier plane wave spectrum propagates along the z-axis (imaging axis) as [7]

$$
\widehat{E}\left(k_{x}, k_{y}, z\right)=\widehat{E}\left(k_{x}, k_{y}, z=0\right) e^{i k_{z} z}
$$

where $k_{z}$ is derived from from the isofrequency relation,

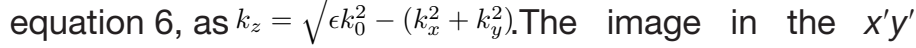
plane (at $z=z>0$ ) then corresponds to the inverse Fourier transform of this spectrum (eq 8)

$$
\begin{aligned}
& \vec{E}(x, y, z)= \\
& \quad \int_{-\infty}^{+\infty} \widehat{E}\left(k_{x}, k_{y}, z=0\right) e^{i\left(k_{x} x+k_{y} y\right)} e^{i k_{z} z} d k_{x} d k_{y}
\end{aligned}
$$

However, from equation 6 we see that for large $k_{x}$ or $k_{y}$, then $k_{z}$ is complex, and the $e^{i k_{z} z}$ term in equation 9 is exponentially damping; these waves are evanescent and decay before reaching the far-field (fig 5a). That is, the high spatial frequencies necessary to make up the sharp features of the object, do not propagate and therefore cannot be detected nor imaged in the far-field ${ }^{2}$. This is known as the diffraction limit and this concept is illustrated in figure 4.

The maximum magnitude of spatial frequency wavevector allowed to propagate and hence contribute to a far field image is given by equation 6 and is written explicitly as

$$
k_{\max } \leq \sqrt{\epsilon} k_{0}=n k_{0}
$$

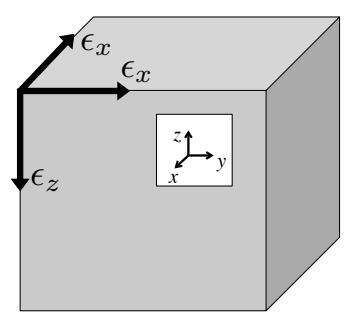

FIG. 2. Schematic of the dielectric tensor for a uniaxial medium. There is a frequencies making up the electric field planar symmetry in the xy-plane so that of an aperture or object in the $\varepsilon_{\mathrm{x}}=\varepsilon_{\|}$, and $\varepsilon_{\mathrm{z}}=\varepsilon_{\perp}$.

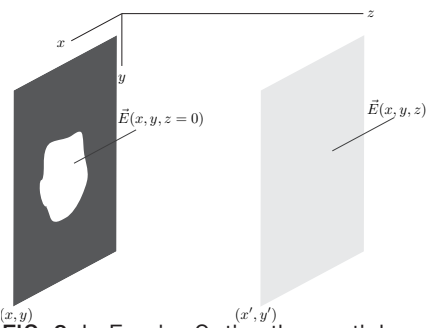

FIG. 3. In Fourier Optics the spatial $(x, y, z=0)$ plane are propagated to the imaging plane $\left(x^{\prime}, y^{\prime}\right)$.
1. For the case of uniaxial crystal propagation, both $\varepsilon_{\|}>0$ and $\varepsilon_{\perp}>0$, so that the wavevector surface is an ellipsoid, and the remaining diffraction limit arguments still apply but must be modified slightly.

2. Far field refers to an image plane with $z>\lambda$ (image field). 


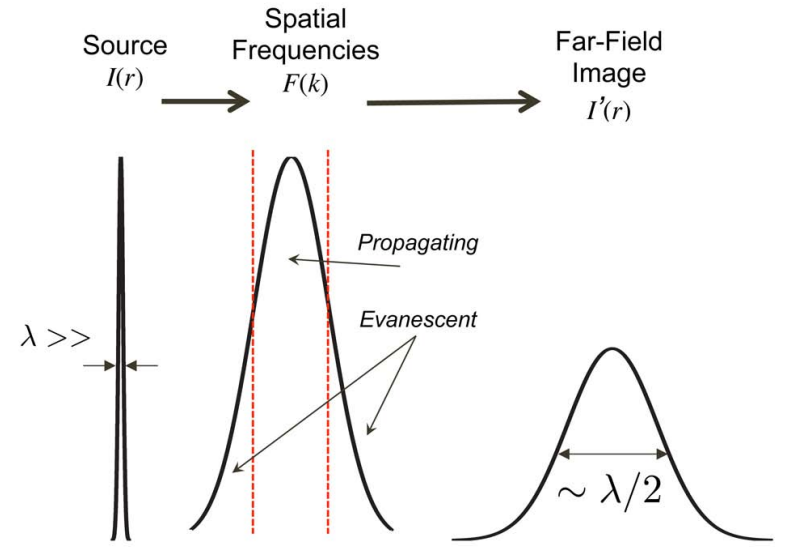

FIG. 4. A highly localized EM source, object, or an object with sharp features has a broad spatial frequency spectrum (fourier spectrum). The high spatial frequencies correspond to evanescent plane waves which decay and do not reach the farfield. As a result, the reconstructed image has lost information, and any original feature smaller than about $\lambda / 2$ is blurred. Furthermore, objects closer than $\sim \lambda / 2$ to each other can not be resolved using traditional optics.

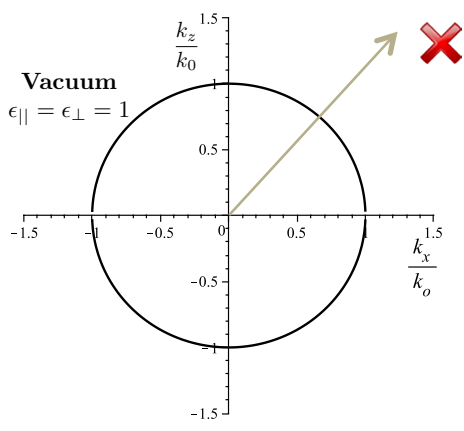

(a) Vacuum

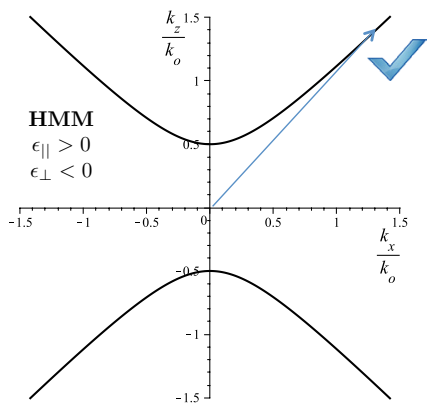

(b) HMM
FIG. 5. (a) In Vacuum and natural dielectric materials, high spatial frequency modes are not supported and are evanescent. High spatial frequencies (large $k_{x}$ ) above $\varepsilon \mathrm{k}_{0}$ yield imaginary propagating wavevectors $\left(\mathrm{k}_{\mathrm{z}}\right)$, and these components of the Fourier (angular) spectrum of an object do not reach the far field (image field). (b) HMMs have a hyperbolic dispersion relation which supports all spatial frequencies. This implies that the whole Fourier or Angular spectrum is propagated to the far field, making HMMs ideal for subwavelength imaging appliations.

This corresponds to a minimum resolvable feature size of $d \approx 2 \pi k_{\max }^{-1}=\lambda / n$. For vacuum, $\mathrm{n} \approx 1.5$ and the minimum resolvable feature size is $\mathrm{d} \approx \lambda / 2$.

\section{Beat the diffraction Limit}

There are several ways to obtain the near field information (high spatial frequencies) of an object such as atomic force microscopy (AFM), or a near field scanning optical microscopy (NSOM). However, these methods are invasive as they require that a detector (usually a very fine tipped needle) be placed very close $(<<\lambda)$ to the object to be imaged [2].Therefore, these sub-diffraction imaging techniques are not suitable for many type of samples.

Other methods of obtaining very high resolution images are scanning electron microscopy (SEM) and transmission electron microscopy (TEM) [2]. These methods utilize the wave nature of electrons (through the de Brogolie relation), and therefore are subject to the diffraction limit as well, however the characteristic wavelength of the electrons can be made much smaller than that of optical and IR light. As a result, the smallest resolvable feature size is smaller than that obtained with conventional optical techniques. However, SEM and TEM tend to be destructive to the sample.

Metematerials provide a new non-destructive/invasive route to allowing these high spatial frequency modes, which normally decay in vacuum and natural materials, to propagate and reach the far field, allowing them to be sensed and imaged.

In the following section, I present a new type of metamaterial, Hyperbolic Metametierials, which allows all spatial frequency modes to propagate into the far field.

\section{HYPERBOLIC METAMATERIALS}

Hyperbolic Metamaterials (HMMs) are an artificially structured material with a dielectric tensor with an extreme anisotropy.

In short, HMMs behave like a metal (with $\varepsilon<0$ ) in one direction and like a dielectric $(\varepsilon>0)$ in another. Planar HMMs are uniaxial and the dielectric tensor has the form

$$
\bar{\epsilon}_{H M M}=\left(\begin{array}{ccc}
\epsilon_{\|} & 0 & 0 \\
0 & \epsilon_{\|} & 0 \\
0 & 0 & \epsilon_{\perp}
\end{array}\right)
$$

with $\varepsilon_{\|}>0$ and $\varepsilon_{\perp}>0$.

This extreme anisotropy yields the exotic behaviour of HMMs: hyperbolic dispersion. With this type of extreme anisotropy, equation 5 yields a wavevector surface of a hyperboloid.

$$
-\frac{k_{x}^{2}+k_{y}^{2}}{\left|\epsilon_{\perp}\right|}+\frac{k_{z}^{2}}{\epsilon_{\| \mid}}=k_{0}^{2}
$$

Using this relation along with the Fourier spectrum arguments made in section II B, all high spatial frequencies are allowed to propagate inside an HMM.That is, for any magnitude of $\sqrt{k_{x}^{2}+k_{y}^{2}}$, there is always a real $k_{z}$, and the whole Fourier or Angular spectrum is propagated into the far field [3]. Therefore, HMMs have a strong potential for sub-wavelength imaging in the far field, that is the ability to beat the so called diffraction limit. The isofrequency curves for an ideal HMM and vacuum are shown in figure 5.

HMMs are not found naturally in nature so must be designed and fabricated to have the required bulk or effective medium properties. Advances in nanofabrication have allowed the nanoscopic structure of materials to be tailored at will, allowing bulk HMM properties to be observed for applications in imaging [4]. In the Effective Medium Theory section below, a novel technique to realize a bulk planar medium with an extreme anisotropy characteristic of HMMs is outlined and discussed.

\section{HMM Design: Effective Medium Theory}

\section{Introduction}

If the structure of an object is much smaller than the wavelength of light interacting with the object, then the response of EM radiation interacting with the structure can be characterized by a bulk or effective medium. Information acquired through measurements on the incident, reflected, and transmitted light will yield properties of an effective, homogeneous medium, not the properties of the media making up the sub-wavelength structure.

Effective medium theory (EMT) or effective medium approximation is a technique to average these subwavelength properties, and predict the bulk response of EM radiation interacting with the structure. Presented in detail 
below is an effective medium theory for planar multilayer structures, and a novel way for designing HMM behaviour in a metal/dielectric multilayer structure.

\section{Effective Medium Theory for Multilayer Structures}

Consider the multilayer structure shown in figure 6 in which the wavelength of the interacting electromagnetic wave $\lambda$ is much larger than the layer thicknesses $d_{1}$ and $d_{2}$. For brevity and simplicity assume $d_{1}=d_{2}=d$. The derivation for the more general case is analogous and will be stated later. The electric flux density in each medium is related to the electric field as

$$
\vec{D}=\bar{\epsilon} \vec{E}
$$

where $\bar{\epsilon}$ is the dielectric tensor. Let each medium on its own be isotropic so that the dielectric tensor can be represented by a dielectric constant, say $\bar{\epsilon} \rightarrow \epsilon_{1}$ for medium 1 and $\bar{\epsilon} \rightarrow \epsilon_{2}$ for medium 2. Therefore, $\overrightarrow{D_{1}}=\epsilon_{1} \overrightarrow{E_{1}}$ and $\overrightarrow{D_{2}}=\epsilon_{2} \overrightarrow{E_{2}}$ Since the wavelength of light is much larger than the layer thickness $\left(\lambda>>\right.$ d) then $\overrightarrow{D_{1}}$ does not vary over several layers of medium 1 , and similarly, $\overrightarrow{D_{2}}$ does not vary over several layers of medium 2,then it follows that the average value of $\vec{D}$ throughout the structure can be determined by analyzing the relation between $\overrightarrow{D_{1}}$ and $\overrightarrow{D_{2}}$ at one interface between medium 1 and medium 2. The average value of $\vec{n}$ will then be related to the average electric field by the effective dielectric tensor

$$
<\vec{D}>=\frac{\vec{D}_{1}+\vec{D}_{2}}{2}=\bar{\epsilon}_{\text {eff }}<\vec{E}>
$$

with $<\vec{E}>=\left(\vec{E}_{1}+\vec{E}_{2}\right) / 2$. The electromagnetic boundary conditions at an interface between medium 1 and medium 2 can be derived from Maxwell's Equations [8]

$$
\begin{aligned}
D_{1}^{\perp} & =D_{2}^{\perp} \\
\vec{E}_{1}^{\|} & =\vec{E}_{2}^{\|}
\end{aligned}
$$

By employing the second of the two above boundary conditions, the average electric flux density parallel to the interface is

$$
\begin{aligned}
<\vec{D}^{\|}> & =\frac{\vec{D}_{1}^{\|}+\vec{D}_{2}^{\|}}{2}=\frac{\epsilon_{1} \vec{E}_{1}^{\|}+\epsilon_{2} \vec{E}_{2}^{\|}}{2} \\
& =\frac{\epsilon_{1}+\epsilon_{1}}{2}<\vec{E}^{\|}>
\end{aligned}
$$

where equation 16 implies $\vec{E}_{1}^{\|}=\vec{E}_{2}^{\|}=<\vec{E}^{\|}>$. The parallel component of the effective dielectric tensor is arithmetic mean of the two dielectric constants of the two media.

Similarly, employing the first of the two boundary conditions, the average electric flux density perpendicular to the interface is computed as

$$
\begin{aligned}
<D^{\perp}> & =\frac{D_{1}^{\perp}+D_{2}^{\perp}}{2}=\frac{\epsilon_{1} E_{1}^{\perp}+\epsilon_{2} E_{2}^{\perp}}{2} \\
& =\frac{2 \epsilon_{1} \epsilon_{1}}{\epsilon_{1}+\epsilon_{2}}<E^{\perp}>
\end{aligned}
$$

where equation 15 was used to relate $E_{1}^{\perp}$ and $E_{2}^{\perp}$.

The perpendicular component of the effective dielectric tensor is the harmonic mean of the two dielectric constants of the two media.

Therefore, the effective dielectric tensor is anisotropic and can then be written as

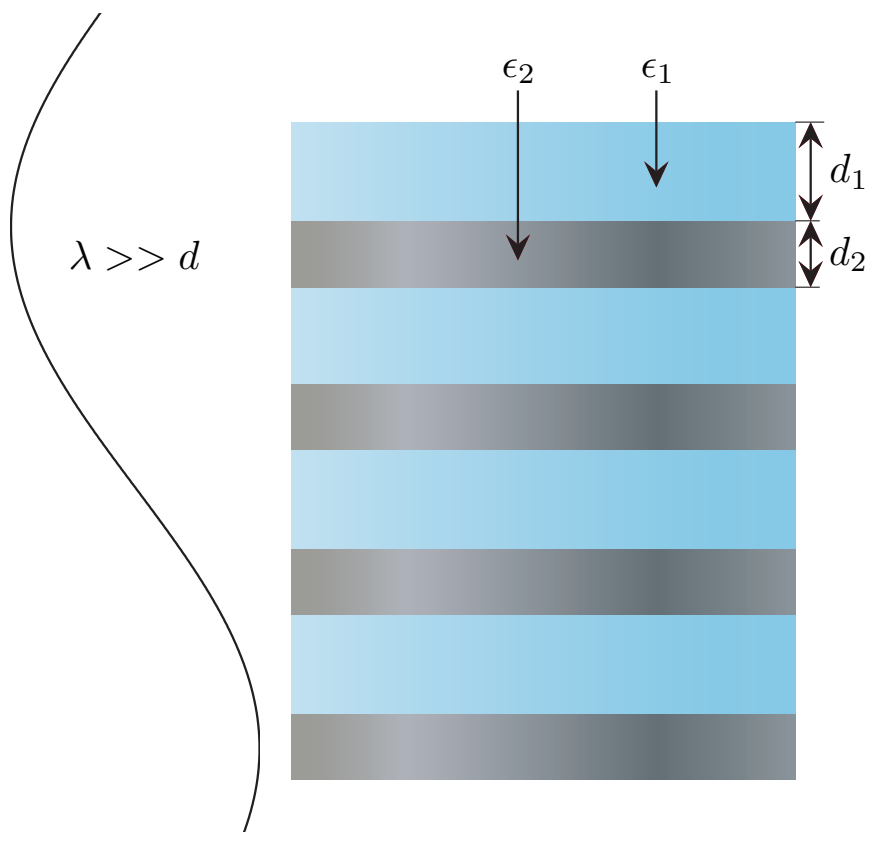

FIG. 6. Multilayer stack used for effective medium theory analysis.

$$
\bar{\epsilon}_{\mathrm{eff}}=\left(\begin{array}{ccc}
\epsilon_{\|} & 0 & 0 \\
0 & \epsilon_{\|} & 0 \\
0 & 0 & \epsilon_{\perp}
\end{array}\right)=\left(\begin{array}{ccc}
\frac{\epsilon_{1}+\epsilon_{1}}{2} & 0 & 0 \\
0 & \frac{\epsilon_{1}+\epsilon_{1}}{2} & 0 \\
0 & 0 & \frac{2 \epsilon_{1} \epsilon_{1}}{\epsilon_{1}+\epsilon_{2}}
\end{array}\right)
$$

where the parallel components lie in the $x y$-plane and the perpendicular direction is considered the $z$-direction.

When $d_{1} \neq d_{2}$, the above can generalized to

$$
\begin{gathered}
\epsilon_{\|}=\rho \epsilon_{2}+(1-\rho) \epsilon_{1} \\
\frac{1}{\epsilon_{\perp}}=\frac{\rho}{\epsilon_{2}}+\frac{1-\rho}{\epsilon_{1}}
\end{gathered}
$$

with the weighting factor $\rho=\frac{d_{2}}{d_{1}+d_{2}}$.

This shows that a general anisotropic uniaxial medium can be fabricated from a periodic multilayer stack with the layer thicknesses much smaller than the desired design EM wavelength. More subtly, however, the thickness of the layers should be chosen so that the quantization of energy levels in the system is irrelevant [5].

\section{Effective Medium Theory For HMM Design}

Referring to figure 6 and equations 20 and 21, if material 1 is a metal and material 2 is a dielectric $\left(\varepsilon_{\|}<0\right.$ and $\left.\varepsilon_{\perp}<0\right)$, then the effective medium dielectric constants are also of opposite signs. That is,

$$
\text { If }\left\{\begin{array} { l } 
{ \epsilon _ { 1 } < 0 } \\
{ \epsilon _ { 2 } > 0 }
\end{array} \text { then } \left\{\begin{array} { l } 
{ \epsilon _ { \| } < 0 } \\
{ \epsilon _ { \perp } > 0 }
\end{array} \text { or } \left\{\begin{array}{l}
\epsilon_{\|}>0 \\
\epsilon_{\perp}<0
\end{array}(22)\right.\right.\right.
$$

which is the exact extreme anisotropy required for HMM behaviour.

Therefore, a simple multilayer structure with alternating layers of dielectric and metal has the bulk EM response required for a HMM.

\section{Design Results: HMM in IR Materials}


For HMM design in the IR region we require a metallic material and a dielectric material to employ the EMT design technique outlined in the previous section.

An obvious choice for the metallic material would be a traditional metal (Ag, Au, etc), however, in the IR losses are much too high. Another possible choice is silicon carbide, $\mathrm{SiC}$, which displays metallic behaviour in the IR [9], however, the dispersion is much too rapid and the losses in the material are significant, making a desired broadband EMT design for imaging applications ineffective. For this design, InGaAs was chosen as it displays metallic behaviour in the IR which can be tailored at will with Si doping. The losses in InGaAs are also relatively low.

As outlined by Hoffman [5], the dielectric constant for InGaAs can be computed using the Drude Model (free electron oscillator) for metals

$$
\epsilon_{\text {InGaAs }}(\omega)=\epsilon_{\infty}\left(1-\frac{\omega_{p}^{2}}{\omega^{2}-i \omega / \gamma}\right)
$$

where $\varepsilon_{\infty}=12.15$ is the background dielectric constant, and $\gamma=0.1 \times 10^{-12} \mathrm{~s}^{-1}$ represents losses in the system and reflects the collision rate of electrons with atoms. The plasma frequency $\omega_{\mathrm{p}}$ can be tailored through Si doping: a donor atom such as phosphorus diffuses into $\mathrm{Si}$ and replaces an Si atom in the regular Si crystal lattice; each donor donates a free electron capable of conducting electricity. If the concentration of donor atoms is $n_{\mathrm{d}}$, then the plasma frequency is given in the Drude model as

$$
\omega_{p}^{2}=\frac{n_{d} e^{2}}{\epsilon_{0} m_{e}^{*}}
$$

where $e$ is the free electron charge, $\varepsilon_{0}$ is the permittivity of free space, and $m_{e}^{*}$ is the effective mass of electrons in InGaAs. Under moderate EM intensities and a doping density of $8 \times 10^{18}$ donors $/ \mathrm{cm}^{3}$, the effective electron mass in InGaAs is $m_{e}^{*} \approx 0.53 m_{e} \quad$ [2] and the plasma frequency is evaluated as $\omega_{p}=2.2 \times 10^{14} \mathrm{~s}^{-1}$

For the dielectric, AllnAs was chosen as it displays very little dispersion in the IR and negligible losses. For most of the IR spectrum $\epsilon_{\mathrm{AIInAs}}=10.23 \quad$. Figure 7 shows the simulated dispersion of the dielectric constants for AllnAs and InGaAs in the IR.

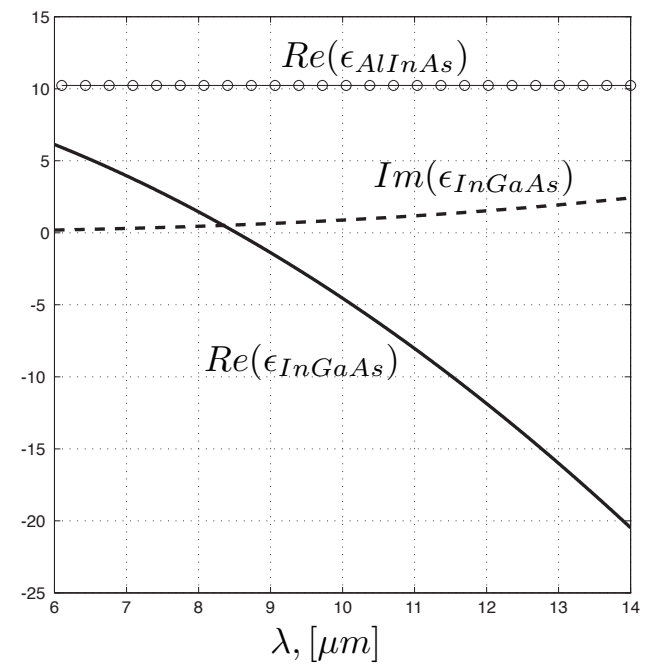

FIG. 7. AllnAs shows very little dispersion in the IR and remains $\approx 10.23$. The real and imaginary parts of $\varepsilon$ InGaAs is computed using the Drude Model for metals at a Si doping level of $8 \times 10^{18} \mathrm{~cm}^{-3}$.
Furthermore, highly crystalline films of these materials can be grown using molecular beam epitaxy.

Since AllnAs/InGaAs are lattice matched, the multilayer structure will have a very low intrinsic stress, and the layer interfaces will be very smooth results in ideal fresnel reflection and transmission [5].

Effective Medium Dielectric Tensor

Using the effective medium design techniques outlined above (equations 20 and 21), along with the material properties derived in the previous section, the effective medium constants $\left(\varepsilon_{\|}\right.$and $\left.\varepsilon_{\perp}\right)$ for a multilayer stack of InGaAs/AllnAs were computed and optimized.

To minimize reflections (with imaging in mind), we require $\varepsilon_{\|} \sim 1$. Therefore, I selected $\varepsilon_{\|}=1$ at a wavelength of $\lambda=10 \mu \mathrm{m}$, roughly the centre wavelength of the desired operation range. In addition, to reduce the total number of layers needed while keeping $\mathrm{d}<<\lambda$, I chose $\mathrm{d}_{\text {AllnAs }}=80 \mathrm{~nm}$. Then from equation 20, the InGaAs thickness is computed as $d_{\text {AllnAs }}=130.5 \mathrm{~nm}$. With reference to equations 20 and 21, this corresponds to a weighting factor $\rho=0.62$.

Using these layer thicknesses, equations 20 and 21, and the dielectric constants of the InGaAs and AllnAs computed in section VA, the effective dielectric tensor was calculated. From this simulation, it was determined that the AllnAs/InGaAs multilayer displayed HMM behaviour in the wavelength region $\$ 8.8-10.5 \mu \mathrm{m}$, for a total bandwidth of $\Delta=1.7 \mu \mathrm{m}$. However, starting $\sim 10.3 \mu \mathrm{m}$ losses begin to become significant, and propagation lengths inside the bulk material (overall multilayer structure) would diminish. Figure 8 shows the wavelength dispersion of $\varepsilon_{\|}$and $\varepsilon_{\perp}$.

\section{Simulation of HMM Behaviour At A Single Spatial Frequency}

The very exotic and useful behaviour of HMMs for applications in sub-wavelength imaging is the fact that normally evanescent modes (high spatial frequencies) are allowed to propagate inside an HMM.

To demonstrate this behaviour, I simulated an evanescent, decaying, plane wave incident upon a slab of HMM (effective medium theory $\varepsilon_{\|}$and $\varepsilon_{\perp}$, and an evanescent wave incident upon the exact multilayer structure $\left(\varepsilon_{\text {InGaAs }}\right.$, $\varepsilon_{\text {AllnAs }}$ ).

Using the fresnel reflection coefficients for TM polarized light incident upon a slab of anisotropic medium (see Fowles [6] for example ), and the expressions for the plane waves developed in section IIA the intensity of an evanescent plane wave $(\lambda=9.8 \mu \mathrm{m})$ incident upon a slab HMM with the effective medium constants shown in figure $\backslash$ ref\{HMMDispersion\} was modeled. The simulation confirmed the expected bulk behaviour of the HMM; namely, evanescent waves are captured by the HMM and are allowed to propagate into the far field. Figure 9 illustrates this result. The figure shows the field profile as the decaying wave propagates in the $\mathrm{z}^{+}$-direction; the $\mathrm{x}$ and $y$-directions have been supressed. We see nearly a 10X increase in the signal strength at the exit side of the HMM slab as compared to vacuum.

To test the validity of the effective medium design and simulation techniques used, a plane wave incident upon the exact InGasAs/AllnAs multilayer structure was modelled. 


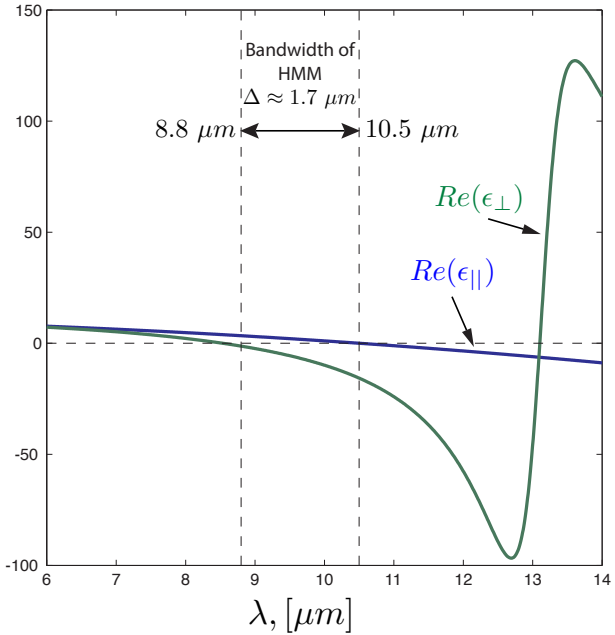

FIG. 8. Dispersion of effective dielectric constants, $\varepsilon_{\|}$ and $\varepsilon_{\perp}$ for AllnAs/InGaAs $(80 \mathrm{~nm} / 130.5 \mathrm{~nm})$ multilayer structure. Multilayer has HMM behaviour when $\varepsilon_{\|}>0$ and $\varepsilon_{\perp}<0(8.8-10.5 \mu \mathrm{m})$.

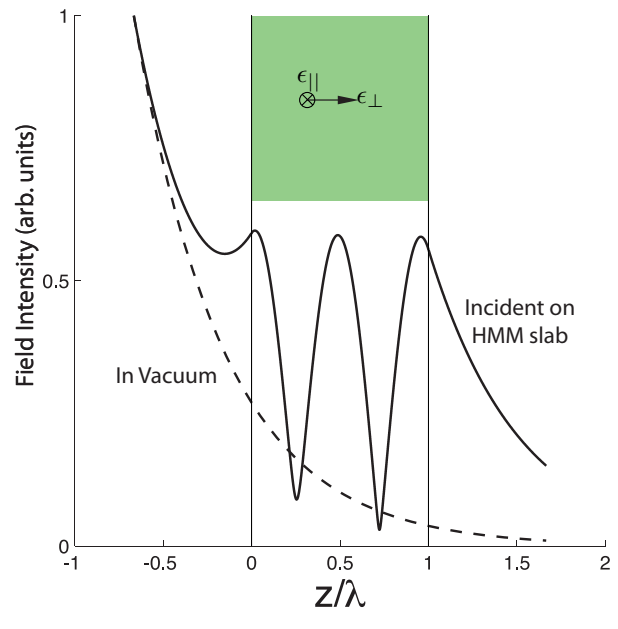

FIG. 9. Evanescent, high spatial frequency waves which decay in vacuum are captured by the HMM slab and allowed to propagate to the far field.

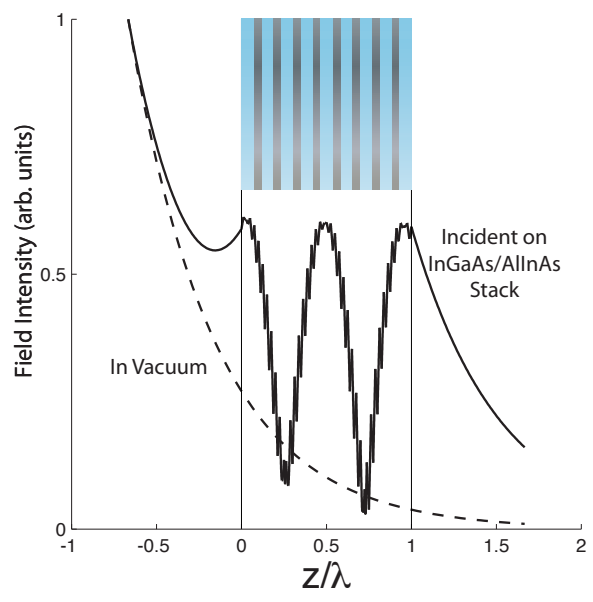

FIG. 10. Exact numerical simulation of the AllnAs/ InGaAs multilayer structure. The bulk response of the multilayer structure agrees very closely with that predicted by effective medium theory. The evanescent, high spatial frequencies waves propagate to the far field through the multilayer structure.
Using a transfer matrix method for obtaining the propagation amplitude constants within multilayer structures (see Yeh [10] for for derivation and discussion of transfer matrix technique), and the form of the fields for plane waves developed in section IIA, the intensity of an evanescent plane wave incident upon the designed AllnAs/ InGaAs multilayer stack was simulated. As shown in figure 10 , the simulation shows the fact that although there is very subtle physics governing the individual layers ${ }^{3}$, the bulk response of the multilayer structure agrees very closely with that observed for the effective medium slab, therefore confirming the validity and accuracy of the effective medium technique for designing HMMs.

We now have confirmation that the multilayer of InGaAs/ AllnAs does posses the exotic HMM behaviour required for sub-diffraction imaging. Below, I show that sub-wavelength resolution of a radiating point source such as an excited $\mathrm{C}-\mathrm{O}$ bond can be obtained in the far-field by utilizing a InGaAs/AllnAs metameterial.

\section{Green's Function Formalism of Scattering and Emission: Sub-wavelength Imaging Introduction}

Scattering, diffraction, and emission centers can be modelled using a radiating point dipole. The details of the near-field (the propagating and evanescent modes), must be obtained through the Green's function formalism of EM sources.

To demonstrate sub-wavelength imaging, I simulated the emitted electric field intensity of a point dipole above a slab of the designed effective medium AllnAs/InGaAs HMM.

3. The high $k_{x}$ evanescent waves excite surface plasmons polaritons on the metal layers, a collective oscillation of electrons within a metallic layer. These surface plasmons then couple together between metallic layers to yield a collective response and allow the evanescent wave to propagate. See Maier's book for an in depth discussion of this phenomena[11].

\section{Derivation and Results}

If there is a localized current source $\vec{J}(\vec{r})$ in vacuum then if we assume time harmonic fields (i.e. all fields and the current source oscillate in time at a frequency $\omega$ ), Maxwell's wave equation for the resulting electric field becomes

$$
\left[(\nabla \times \nabla \times)-k_{0}^{2}\right] \vec{E}=i \frac{4 \pi k_{0}}{c} \vec{J}
$$

again with $k_{0}=\omega / c$. This is the well known vector Helmholtz equation.

Now, a point dipole located at the position $\vec{r}=\vec{r}_{0}$ can be described by a point current source $\vec{J}(\vec{r})=\vec{\mu} \delta\left(\vec{r}-\vec{r}_{0}\right.$ where $\vec{\mu}$ gives the direction of the dipole (current source).

With this point current source, Maxwell's wave equation can be solved using a Green's Function Technique [7].

The derivation is quite in depth and only the results will be presented here. For simplicity, the dipole is oriented normal to the slab; in this way, all the EM radiation incident on the HMM is TM polarized [7]. By employing the Green's Function Technique for solving the vector Helmholtz equation, the general form for the fields emitted by a radiating point dipole oriented along the $z$-axis $(\vec{\mu}=[0 ; 0 ; 1])$ and at a position $z=d$ can be shown to be

$$
\vec{E}=\left[\widehat{D} \iint_{-\infty}^{+\infty} \frac{1}{k_{z}} e^{i\left(k_{x} x+k_{y} y+k_{z}|z-d|\right)} d k_{x} d k_{y}\right] \cdot\left(\begin{array}{l}
0 \\
0 \\
1
\end{array}\right)
$$

where the differential operator $\widehat{D}$ being

$$
\widehat{D}=\left(\bar{U}+\frac{1}{k_{0}^{2}} \vec{\nabla} \vec{\nabla}\right)
$$

and

$$
\bar{U}=\left(\begin{array}{lll}
1 & 0 & 0 \\
0 & 1 & 0 \\
0 & 0 & 1
\end{array}\right)
$$

The double integral term represents the angular spectrum or plane wave representation of a scalar point source emitting spherical waves.

The Dyadic operator $\widehat{D}$, acting on this integral essentially 
vectorizes and transforms the spherical point source into electric fields corresponding to the orientation of the dipole vector $\vec{\mu}=(0 ; 0 ; 1)$.

The double integral in equation 26 is very computationally heavy due to the limits of integration, and moreover, because there is a singularity when $k_{z}$ changes from real to imaginary (when the spatial frequencies become evanescent).

It is numerically convenient to then convert this 2-D integral which ranges over $\left(k_{x}, k_{y}\right)$ into a 1-D integral which runs over $k_{r}$ through a cylindrical coordinate transform, and in addition, to split the integrals up into propagating and evanescent parts [12]. The forms of the decomposed integrals are then the well known numerical "Sommerfeld Integrals".

Because the form of the double integral in equation 26 is that of a summation of plane waves $\sim e^{i\left(k_{x} x+k_{y} y+k_{z} z\right)}$ with amplitudes $1 / k_{z}$ then it is possible to obtain the forms of the reflected and transmitted electric fields, $\vec{E}_{R}$ a $\mathrm{n} \mathrm{d} \vec{E}_{T}$ using the fresnel reflection and transmission coefficients at a single spatial frequency pair $\left(k_{x}, k_{y}\right)$. Similarly, the resulting integrals for $\vec{E}_{R}$ and $\vec{E}_{T}$ can then be converted to Sommerfeld integrals.

Using the tools derived above, the fields of a monochromatic radiating dipole $(\lambda=9.8 \mu \mathrm{m})$ were then simulated in MATLAB for two cases.

First, when the dipole was isolated in vacuum, and second when the dipole was brought near the effective medium HMM of the InGaAs/AllnAs multilayer.

The results of these simulations are shown in figure 11.

In vacuum the high spatial frequency modes required for imaging the very small features of the source decay quickly and the over all emission is essentially non-directional. As the source is brought close to the HMM, the high spatial frequency modes couple into the HMM resulting in highly directional emission. This very important result shows that very high resolution information may be carried to the farfield. The implications of this extremely exotic behaviour are discussed in the section below.

\section{Device Implications and Design}

The planar HMM designed in this project has a wide range of applications from a bio-analytical tools to integrated circuit quality control.

Each chemical species emits a unique IR fingerprint due to characteristics of the vibrations in the chemical bonds. This allows for spectroscopy to identify specific chemical
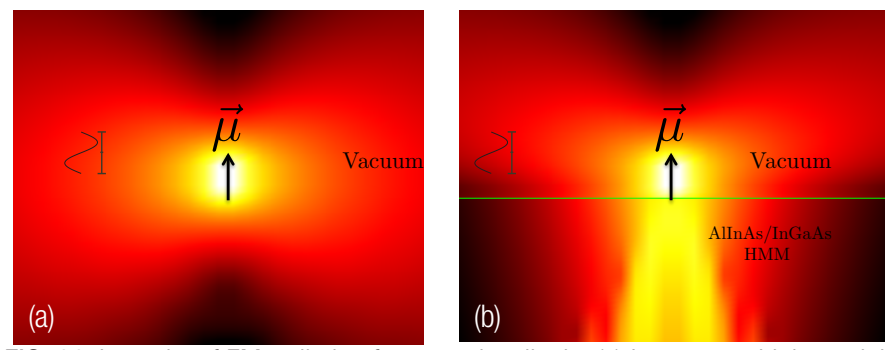

FIG. 11. Intensity of EM radiation from a point-dipole. (a) In vacuum, high spatial frequency modes decay and emission is non-directional. (b) When dipole is brought near HMM, the high spatial frequency, evanescent modes couple into HMM resulting in highly directional emission. High spatial frequencies reach far field. species, based on the observed IR spectrum.Combining spectroscopy with microscopy allows for drug permeation in human tissue to be observed (fig 12).

By using HMM sub-wavelength imaging techniques, it may be possible to observe drug permeation and chemical activity within a single cell. The characteristic size of cells is much smaller than that of tissue, so to resolve these sub-wavelength (IR) features, sub-wavelength imaging techniques are required. This type of application has incredible importance especially in the pharmaceutical industry, and in the Bio-MEMs industry.

$\mathrm{Si}$ is transparent to IR wavelengths; therefore, cracks or defects in ICs or MEMs can be detected through the scattering and diffraction of IR light at these defect centres [14]. The main problem with the conventional technique is the diffraction limit: cracks and defects smaller than the illuminating wavelength can not be imaged. In terms of IR this corresponds to $\sim 2-3 \mu \mathrm{m}$. By using a HMM, subwavelength resolution may be obtained so that defects with sub-wavelength dimensions may be observed and detected. This type of application/ device is conceptualized in figure 13.

\section{Conclusion}

I have motivated further research in hyperbolic metamaterials as a means to beating the diffraction limit and presented a proof of concept of a semiconductor based device capable of sub-diffraction and sub-wavelength

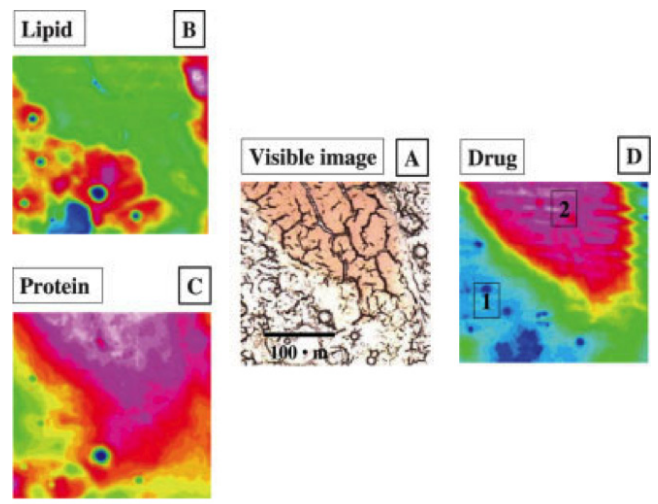

FIG. 12. Shown: utilizing spectroscopy and microscopy allows for drug permeation within tissue to be observed. Figure from [13]. By employing sub-wavelength imaging techniques with HMMs in combination with spectroscopy, drug permeation within individual cells (cell dimensions are much smaller than that of tissue) may be obtained (nano-spectroscopy).
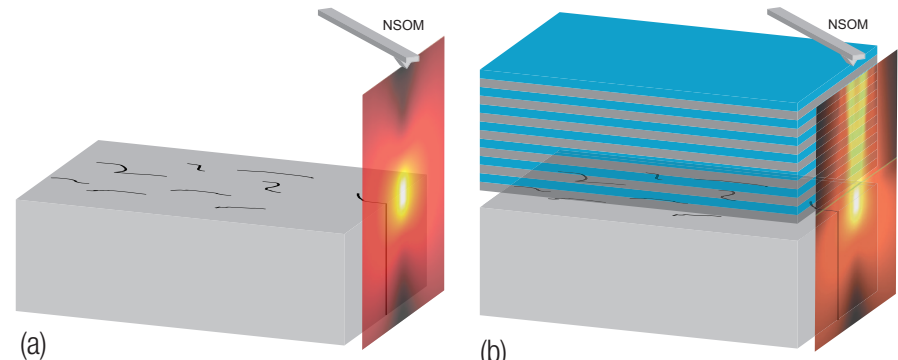

(b)

FIG. 13. Illumination of a Si wafer from below by $\lambda=9.8 \mu \mathrm{m}$ light. The incident light scatters through cracks in the wafer. (a) A sub-wavelength dimension crack in a Si wafer can be detected by a near field scanning optical microscope (NSOM) only if the detector is brought very close to the crack $(<\lambda / 6)$ (b) If a AllnAs/lnGaAs HMM is placed above the wafer, then the NSOM can detect the crack up to $2-3 \times \lambda$ from the wafer. A $10 \times$ increase in far field enhancement of high spatial frequencies can be acheived. 
imaging.

The necessary theory and electrodynamic formalism for describing the diffraction limit and sub-wavelength imaging was presented. Classical electrodynamics and the concept of Fourier optics was used to explain the conventional diffraction limit. Hyperbolic metamaterials, which have a hyperbolic dispersion and allow high spatial frequencies to propagate, were proposed as a solution to overcoming the diffraction limit.

Effective medium theory was developed for periodic multilayer structures and put forth as a design tool for hyperbolic metamaterials. It was shown that a multilayer stack of alternating layers of metal/dielectric displays hyperbolic metamaterial behaviour with a bulk extreme anisotropy: it behaves like a dielectric in one direction and a metal in another.

A hyperbolic metamaterial was then designed and demonstrated in the IR. Using a multilayer structure of AllnAs/ InGaAs (thickness 80/130.5 nm), with AllnAs as a dielectric and InGaAs as a customizable metal, it was shown that this multilayer structure exhibits bulk hyperbolic metamaterial behaviour in the IR region of wavlengths $\lambda=8.8$ to 10.5 $\mu \mathrm{m}$. Furthermore, the novel and exotic behaviour of the hyperbolic metamaterials was demonstrated: hyperbolic metamaterials capture evanescent near field waves and allow them to propagate to the far field. This was simulated for the effective medium slab of hyperbolic metamaterial and on the exact AllnAs/InGaAs multilayer structure using a transfer matrix technique. It was demonstrated that the bulk response of the exact multilayer model agrees very closely with that of the effective medium theory, therefore confirming the validity of the effective medium theory design technique for planar hyperbolic metamaterials.

The most difficult and also most important portion of the design was then completed. Namely, sub-wavelength imaging capabilities were demonstrated by showing a highly directional emission into the far field from a radiating point source near the effective medium AllnAs/InGaAs structure. The intricate and subtle details of the electric fields emitted by the point source was modeled through the Green's function formalism of EM sources. Potential devices and applications in pharmaceuticals and IC quality control were then proposed for this planar AllnAs/InGaAs hyperbolic metamaterial.

Future work will involve completing the last confirmation of the design: demonstrate sub-wavelength imaging capabilities by simulating the behaviour of a radiating point source above the exact multilayer structure. Moreover, experimental evidence must be obtained by fabricating and characterizing the AllnAs/InGaAs multilayer using molecular beam epitaxy.

\section{Acknowledgements}

This work was completed as a fourth year, Capstone Design Project in the department of Electrical and Computer Engineering. I would like to thank Dr. Chris Backhouse for instructing the course and providing useful feedback and direction throughout the course. My supervisor for the project was Dr. Zubin Jacob, an excellent teacher. Dr. Jacob provided the invaluable support and instruction required for understanding the complex electrodynamics and for me to enter into the field of metamaterials. In addition, I would like to thank my colleague, Sean Molesky for numerous insightful discussions.

\section{Reference}

[1] E. Hecht. Optics. Addison-Wesley, fourth edition, 2002.

[2] S. A. Campbell. Fabrication engineering at the micro and nanoscale. Oxford University Press, 2008.

[3] Z. Jacob, L. V. Alekseyev, and E. Narimanov. Optical Hyperlens: Far-field imaging beyond the diffraction limit. Optics express, 14(18):8247-56, September 2006.

[4] Z. Liu, H. Lee, Y. Xiong, C. Sun, nd X. Zhang. Far-field optical hyperlens magnifying sub-diffraction-limited objects. Science (New York, N.Y.), 315(5819):1686, March 2007.

[5] A. J. Hoffman, L. Alekseyev, S. S. Howard, K. J. Franz, D. Wasserman, V. a Podolskiy, E. E. Narimanov, D. L. Sivco, and C. Gmachl. Negative refraction in semiconductor metamaterials. Nature materials, 6(12):946-50, December 2007.

[6] G. R. Fowles. Introduction to Modern Optics. Dover, 1989.

[7] L. Novotny and B. Hecht. Principles of Nano-Optics, volume 54. Cambridge Univ Press, 2006.

[8] J. D. Jackson. Classical Electrodynamics. Wiley, third edition, 2007.

[9] T. Taubner, D. Korobkin, Y. Urzhumov, G. Shvets, and R. Hillenbrand. Nearfield microscopy through a SiC superlens. Science, 313(5793):1595, September 2006.

[10] P. Yeh. Optical Waves In Layered Media. Wiley, New York, 1988.

[11] S. A. Maier. Plasmonics (Fundamentals and Appli-cations). Springer, New York, 2007.

[12] T. Setala, M . Kaivola, and A. T. Friberg. Decomposition of the point-dipole field into homogeneous and evanescent parts. Physical Review, 59(1):1200-1206, May 1999.

[13] P. Garidel and M. Boese, Mid infrared microspectroscopic mapping and imaging: A bio-analytical tool for spatially and chemically resolved tissue characterization and evaluationof drug permeation within tissues. Microscopy Research and Technique, 70(4):336-349, 2007.

[14] A. Trigg. Applications of infrared microscopy to IC and MEMS packaging. IEEE Transactions on Electronics Packaging Manufacturing, 26(3):232-238, July 2003. 


\section{METAMATERIAL ENHANCED FLUORESCENCE DETECTION}

Sean J. Molesky

Electrical and Computer Engineering, University of Alberta

In this article I show how materials created from designer functional units much smaller than the wavelength of operation , or metamaterials, can be used to decrease the lifetime of fluorescence based emitters. This is goal accomplished in three parts. First, the fundamental physical equations describing both fluorescent emission and the particular class of metamaterial required will be over viewed in a broad two part introduction. Next, making use of a seldom seen approach, I will present the Green's functions formalism of spontaneous emission of a quantum emitter above a material slab. Finally, I will present devices to reduce the lifetime of quantum emitters that could provide a large resolution enhancement for fluorescence based sensors, focussing on the wavelengths of 600 and $800 \mathrm{~nm}$.

\section{INTRODUCTION AND MOTIVATION}

Considering the ubiquity of quantum emitters in both device application and understanding throughout all branches of the natural sciences, it should not be surprising to learn just how much research has gone into the prospect manipulating their behavior in the hopes of producing superior device performance [1, 2]. Yet, despite the best efforts of the intellectual giants of the field, until incredibly recently a satisfactory method for significant broadband manipulation of emitter lifetime, the key parameter governing the properties of quantum emitters, had not been found [3].

To gain a better understanding of what this parameters means, we begin by recalling Fermi's golden rule:

$$
R_{s}=\frac{2 \pi}{\hbar}\left|\left\langle\Psi_{f}\left|H_{\text {int }}\right| \Psi i\right\rangle\right|^{2} n\left(\epsilon_{f}\right)
$$

Where $\Psi$ represents the intial quantum state, $n\left(\epsilon_{f}\right)$ the final density of states and $\mathrm{H}$ the Hamiltonian of the system [4].

This equation allows us to see that the lifetime of an excited state relies heavily on the final density of states which the quanta of energy may occupy. For fluorophores, the examples of interest in this article, this means the lifetime of the excited vibrational state is determined by the states that the released photon could possibly occupy upon emission. Or, from a perhaps more instinctively strange anthropomorphic perspective, a photon is much more likely to come into being if its feelers find many places where could fit in. With this somewhat odd reflection in mind, engineering the lifetime of fluorophores, or any other quantum emitter, is a matter of altering the number of states a photon of a given frequency could exist in, or more concisely the photonic density of states.

Conventionally, the primary means for producing the desired enhancement to the photonic density of states has

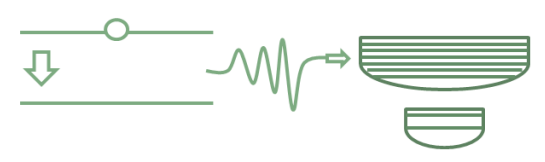

Fig. 1 Lifetime of the Excited State is Based on the Density of States Available to the Emission

Fig. 2 Cavity to Reduce Emitter Lifetime Via the Purcell Effect Michler et. al been through the use of resonator cavities, based on the ingenious work of noble prize winning physicist Edward Purcell. While perhaps overly simplistic, the effect bearing Purcell's name can be understood almost entirely from considering an argument about emitted power ${ }^{1}$ :

Given that it is possible to couple power from a photon emitter into the resonant modes of a cavity above and beyond what is possible in free space, provided there is an overlap between the bandwidth of the emission and that of the resonant mode [5]. In equal measure, it makes intuitive sense that an increase in the net power output must be directly tied to a reduction of the lifetime of any excited state from which photon emission occurs. Therefore, resonant cavity modes must cause an enhancement to the photonic density of states, and in turn offer an engineering solution to achieving control over devices based on quantum emitter .

Unfortunately, in practical application the resonant cavity approach is severally limited by the typically minuet bandwidth of resonant cavity modes in comparison to that of room temperature photonic emitters [6]. In order to produce a meaningful enhancement of the photonic density of states, required to get the best possible results from sensing and detection based devices, a new approach must be taken. This is no easy task in view of the possibilities presented by standard materials.

However, recent results suggest that this does not mean a new solution to broadband augmentation of the photonic density of states is not possible. The maturation of nanostructure fabrication techniques has turned many fantasies into practical standard recipes, and its a good guess to assume that once again it will play a leading role [7]. Using arrays of designer nanostructures, metamaterials with properties above and beyond anything found in nature can now be produced and functionally implemented with a high degree of precision; creating a new paradigm for a host of long standing engineering problems. As it currently appears, the problem of how to alter the photonic density of states can certainly be included in this category.

\section{UNDERSTANDING HYPERBOLIC METAMATERIALS Isofrequency Surfaces and Maxwell's Equations \\ Perhaps the most efficient way to understanding the hyperbolic metamaterial solution to enlarging the photonic density of states is to return to the fundamental electromagnetic properties necessitated by Maxwell's}

\footnotetext{
1. A thorough derivation of the Purcell effect is fairly involved and will not be dealt with in this article.
} 
equations [8]:

$$
\begin{gathered}
\vec{\nabla} \cdot \vec{D}=\rho_{f} \\
\vec{\nabla} \times \vec{E}=-\frac{\partial \vec{B}}{\partial t} \\
\vec{\nabla} \cdot \vec{B}=0 \\
\vec{\nabla} \times \vec{H}=\vec{J}_{f}+\frac{\partial \vec{D}}{\partial t}
\end{gathered}
$$

Assumming plane wave solutions of the form:

$$
E(\vec{r}, t)=A_{0} \operatorname{Re}\left(e^{i(\vec{k} \cdot \vec{r}-\omega t)}\right)
$$

Where $\mathrm{k}$ the wave vector with a magnitude defined by $\frac{2 \pi}{\lambda}$.

Taking a simple approach, this quantity can be understood as a measure which is inversely proportional to wavelength that dictates the propagation direction of the plane wave by (6). If the curl of (5) is taken, in conjunction with the result of (3), in a linearly polarisable material free of charges and currents it can be found that:

$$
\frac{k_{x}^{2}}{\epsilon}+\frac{k_{y}^{2}}{\epsilon}+\frac{k_{z}^{2}}{\epsilon}=\frac{\omega^{2}}{c}
$$

Which, if the frequency is fixed, has a form equivalent to the mathematical description of a sphere.

We can now easily develop a graphical understanding of the photonic density of states. Given that each possible photonic state can be represented in wave vector space as a small cube of some dimension, dictated by the properties of the material in which the surface is plotted, the photonic density of states can be pictured as the amount of volume that would be added if the sphere were given an infinitesimal thickness, and turned into a shell, through an infinitesimal addition to the band of its frequency (7). This strictly geometric interpretation depicts a number of very important realizations. In particular, it shows the number of photonic states must be fixed as the volume of an added shell is always finite ${ }^{2}$.

\section{Hyperbolic Metamaterials}

Bearing this in mind, we can now begin to consider more complicated scenarios. If the dielectric constant of (7) were directional and of undefined sign, then (7), following the treatment descried in the beginning of this section, would be transformed into

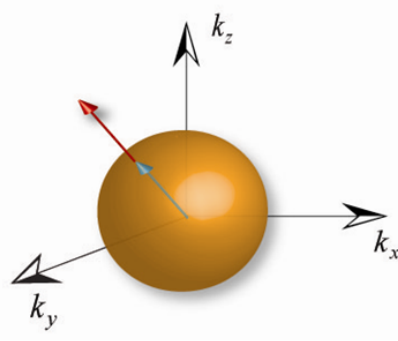

Fig 3. Isofrequency Surface of the Free Space Dispersion Relation

$$
\frac{k_{x}^{2}}{\epsilon_{x}} \pm \frac{k_{y}^{2}}{\epsilon_{y}} \pm \frac{k_{z}^{2}}{\epsilon_{z}}=\frac{\omega^{2}}{c}
$$

Which serves as the generating equation for a vast range of conic surfaces. In particular, if in two directions the dielectric were of positive sign and in the other negative, technically known as an extreme anisotropy in the dielectric tensor ${ }^{3}$, the relation of a hyperboloid would result. Returning briefly to the graphical scheme presented earlier in this section, if such a dispersion relation were possible then an unbounded density of states would be produced by virtue of the unbounded wave vector magnitude describe by:

$$
\frac{k_{x}^{2}}{\epsilon_{\perp}}+\frac{k_{y}^{2}}{\epsilon_{\perp}}-\frac{k_{z}^{2}}{\epsilon_{\|}}=\frac{\omega^{2}}{c}
$$

As has recently been explored in the context of creating a hyperlens [9], a certain class of metamaterials, known as hyperbolic metamaterials, are capable of displaying

this exact behaviour. With a reasonable concept of the basis of the metamaterial solution firmly in place, our attention may now turn to how such materials may be fabricated, and a more rigorous description of their exact effects on photonic emitters.

\section{Effective Medium Theory}

On the macroscopic scale, the means for producing hyperbolic metamaterials, and thus gaining improved control over the decay rate of fluorophores, is strongly tethered to the averaging procedures ${ }^{4}$ which describes the reaction of an electromagnetic wave to sub wavelength features. If we consider a stack of materials with dielectric constants of alternating magnitude, it is not difficult to show that the relations imposed by Maxwell's equations (2) between the electric flux density, $\vec{D}$, and fields, $\vec{E}$, of the alternating slabs are given by:

$$
\begin{aligned}
\vec{D}_{1 / 2} & =\epsilon_{1} \vec{E}_{1 / 2} \\
E_{1}^{\|} & =E_{2}^{\|}
\end{aligned}
$$

2. The diffraction limit arises as the maximum magnitude of the wave vector, for propagating waves, is bound by the isofrequency sphere. Were one of the wave vector components larger than the fixed radius dictated by (7) one of the other two wave vector components would have to be imaginary, resulting in a decaying or evanescent wave through (6). Isofrequency surfaces may also be used to show why emission in hyperbolic metamaterial must be directional. As energy must always flow in the direction of group propagation, which through the gradient is always perpendicular to the iso-surface, for any particular hyperbolic metamaterial there will be a perferred direction for energy flow.

3. A tensor is an array of the second rank which maps the directionality of the dielectric into a form that can be used in conjunction with Maxwell's equations (2-5).

4. For objects much smaller than the wavelength of light at a given frequency the bulk or macroscopic behavior can be well modeled by assuming the small objects form an effective medium. One of the more popular procedures for determining the parameters of this medium known as the Maxwell-Garnett theory. 


$$
D_{1}^{\perp}=D_{2}^{\perp}
$$

Which, if the wavelength of the electromagnetic perturbation is sufficiently large in comparison to the thickness of a few layers, such that it may be assumed that $\vec{D}$ and remain constant over the interval, give rise to the effective media descriptions:

$$
\begin{gathered}
\epsilon^{\|}=\epsilon_{1}(P)+\epsilon_{2}(1-P) \\
\frac{1}{\epsilon^{\perp}}=\frac{P}{\epsilon_{1}}+\frac{(1-P)}{\epsilon_{2}}
\end{gathered}
$$

Where $\mathrm{P}$ represents a fill factor based on the proportion of one of the two alternating slabs to the other, $\frac{d 1}{d 1+d 2}$.

Looking carefully at these equations and recalling the result of (8), it can be seen that if one of the dielectrics is given a positive magnitude, which in the context of dielectrics will be referred to as a dielectric, and the other a negative magnitude, characteristic of metals, that the desired hyperbolic dispersion relation would result. Despite the seeming superficiality of this argument, the result is quite accurate provided that all of the assumptions made in its construction are satisfied. To implement a hyperbolic metamaterial all that is needed is defined layers of alternating metal and dielectric of the correct thickness.

\section{Surface Plasmon Polariton (SPP)}

While the effective medium approach is an extremely useful description of the macroscopic characteristics of a hyperbolic metamaterial (HMM), it provides little explanation of how these phenomena arrive from the nanosized building blocks. Thus, in order to have a firm enough grasp of the concepts involved in creating HMM to move on to a satisfactory exploration of their effects on fluorophores it is crucial to also understand the surface plasmon polariton state.

Directly, a surface plasmon polariton (SPP) mode is as quanta of energy trapped in an oscillatory mode which couples the free electron density at a surface to incoming photons. Or, in turning to the mathematical sophistication of Maxwell's equations, as a TM electromagnetic boundary wave solution described by:

$$
k_{x}=\frac{\omega}{c}\left(\frac{\epsilon_{1} \epsilon_{2}}{\epsilon_{1}+\epsilon_{2}}\right)^{1 / 2}
$$

(Found by guessing at the existence of a state characterized by a magnetic field perpendicular to the plane and an electric field in the other two perpendicular directions ${ }^{5}$ and an application of the boundary conditions from Maxwell's equations. $)^{6}(10)(11)$

While the general shape and field confinement of the SPP solution is interesting in and of itself, it is in applying the Drude model:

$$
\epsilon_{2}(\omega)=1-\omega_{p}^{2} / \omega^{2}
$$

where $\omega_{p}$ is a material property known as the plasma frequency, (8) to (15) that truly exciting behaviour occurs.

From the preceding plot of the SPP dispersion relation it can be seen that at the frequency $\frac{\omega_{p}}{\sqrt{2}}$ the over all behaviour of wave vector frequency relation becomes

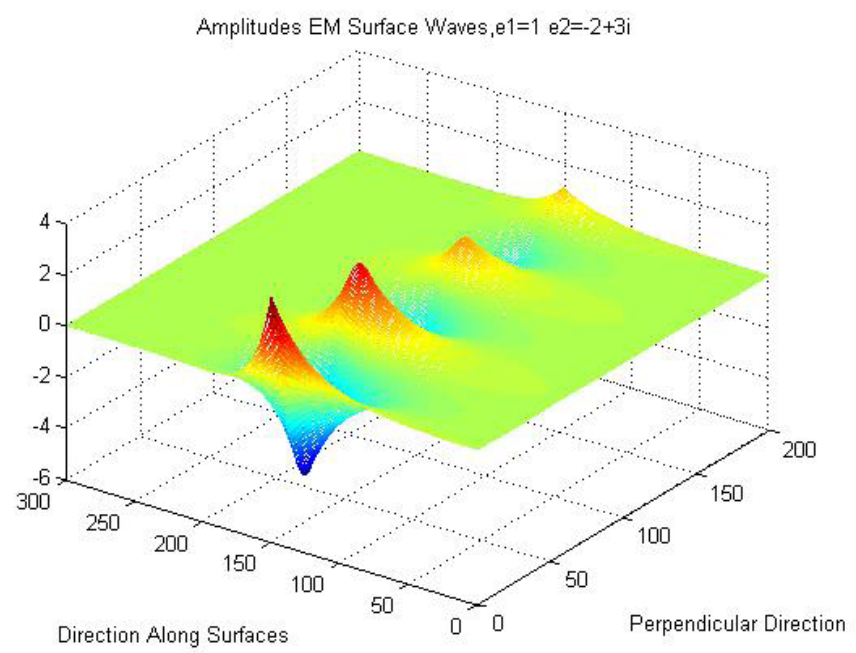

Fig. 5. Plasmon Polaritron for Lossy Metal

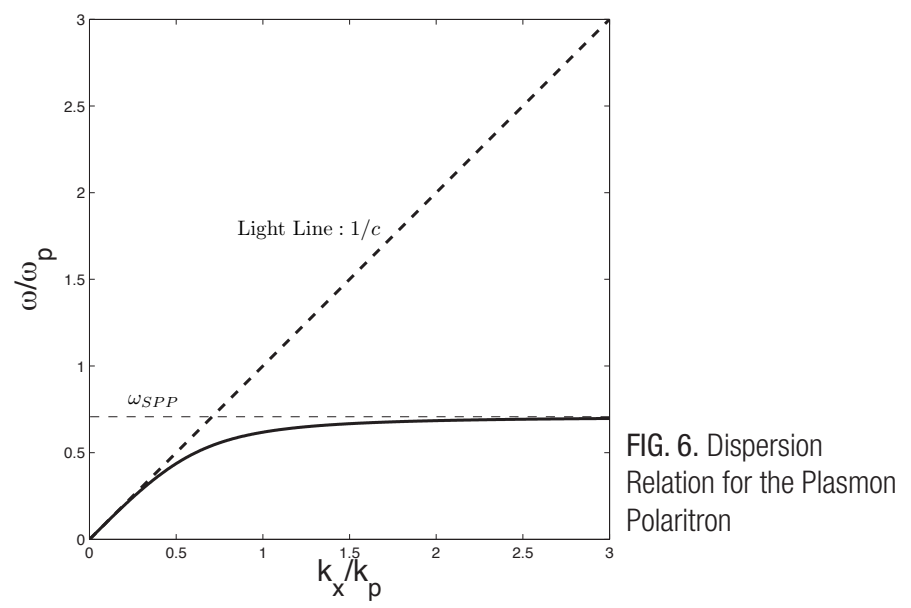

asymptotic; meaning that a photon coupled to the free electron density at the surface of a metal could once again access an essentially unlimited number of states by virtue of the unbounded magnitude of its wave vector. This is no coincidence, it turns out that through coupling to the SPP modes which exist simultaneously at each metal dielectric interface that the bulk properties of a HMM can be recovered. As these views are inherently interreliant, this consideration also gives an inkling to which metals are best suited for hyperbolic metamaterial implementations ${ }^{7}$. We are now ready to pursue the mathematical treatment required to more fully comprehend fluorophore lifetime in the presence of hyperbolic metamaterials.

\section{MATHEMATICAL TREATMENT FOR CALCULATING LIFETIME NEAR METAMATERIALS}

Green's Function Method

We will now derive a rather novel Green's function

5. Electromagnetic fields with this type of orientation are more commonly referred to as transverse magnetic, or TM solutions.

6. Historically, the idea of a SPP was introduced by Zenneck and Sommerfeld to acount to the extra energy observed in radio transimissions compared to the expected free space values.

7. Based off this discussion it would seem that any metal with a strong SPP mode would be a good candidate.

8. The Green's function method for solving differential equations is based on the response of a system to a point source. This solution can then be moved and weighted to solve the problem for more complicated sources. 
approach to determine the lifetime of a quantum emitter, building on two primary considerations. First, as the Green's function method 8 of solving partial differential equations is at its deepest level a complete description of the correlation between the sources and responses of a given system; a more thorough understanding of the underlying physics of a problem can generally be gleamed through the Green's Function solution. Secondly, a proper mathematical description of the emitted power requires that we consider free space decaying waves which in general cannot be handled by first order perturbation theory.

\section{General Form}

Through an examination of the Green's function solution to the Helmholtz equation, we will finally assemble the necessary mathematical machinery to reach our final theoretical task of determining fluorophore lifetime above a metamaterial.

$$
\begin{gathered}
\left(\nabla^{2}+k^{2}\right) \psi=\frac{2 m}{\hbar^{2}} V \psi \\
k=\frac{2 m E}{\hbar^{2}} \\
\left(\nabla^{2}+k^{2}\right) g\left(r, r^{\prime}\right)=\delta(r)
\end{gathered}
$$

Now, setting $r^{\prime}=0$ and transforming the problem into wave vector space (q), we get:

$$
\begin{gathered}
g(r)=\iiint \frac{d^{3} q}{(2 \pi)^{3}} g(q) e^{i \vec{q} \cdot \vec{r}} \\
\delta(r)=\iiint \frac{d^{3} q}{(2 \pi)^{3}} e^{i \vec{q} \cdot \vec{r}} \\
\iiint\left(\nabla^{2}+k^{2}\right) \frac{d^{3} q}{(2 \pi)^{3}} g(q) e^{i \vec{q} \cdot \vec{r}}=\iiint \frac{d^{3} q}{(2 \pi)^{3}} g(q) e^{i \vec{q} \cdot \vec{r}}
\end{gathered}
$$

Which must be true for all $r$, so:

$$
g(q)=\frac{1}{k^{2}-q^{2}}
$$

This equation, if we displace the poles from the real axis and make a transformation to polar coordinates, can be solved by making use of Cauchy integral theorem, leading to the famous Lippmann-Schwinger equation:

$$
\begin{gathered}
g(r)=-\frac{e^{i k r}}{4 \pi r} \\
\Psi=\Psi_{o}-\int \frac{e^{i k\left|r-r^{\prime}\right|}}{4 \pi\left|r-r^{\prime}\right|} \frac{2 m}{\hbar^{2}} V\left(r^{\prime}\right) \psi d V^{\prime}
\end{gathered}
$$

Which additionally, by making the ansatz that $\psi_{o}=e^{i \vec{k} \cdot \vec{r}}$, is also the general equation of a scattering potential.

$$
\Psi=\Psi_{o}-\int \frac{e^{i k\left|r-r^{\prime}\right|}}{4 \pi\left|r-r^{\prime}\right|} \frac{2 m}{\hbar^{2}} V\left(r^{\prime}\right) e^{i \vec{k} \cdot \vec{r}} d V^{\prime}
$$

\section{Maxwell's Equations and Green's Functions}

Our next undertaking is to cast Maxwell's equations in the form of the Helmholtz equation in order to make use of the results of the previous subsection. Using the fact that the electromagnetic vector potential [8] may be defined as:

$$
\begin{gathered}
\vec{\nabla} \times \vec{A}=\vec{B}^{\prime} \\
\vec{E}=-\frac{1}{c} \frac{\partial \vec{A}}{\partial t}+\vec{\nabla} \phi
\end{gathered}
$$

Maxwell's equations can be used to create a Helmholtz equation for the vector potential, and in turn the electric field, in linearly polarisable media by taking taking the cross product of (26) and using the results of (5) and (27) in the Lorentz guage leading to:

$$
\nabla^{2} \vec{A}-\frac{\mu \epsilon}{c^{2}} \frac{\partial^{2} \vec{A}}{\partial t^{2}}=\frac{-4 \pi \mu \vec{J}}{c}
$$

Which, in Cartesian coordinates, through the use of the previous subsection and the well known Wyle identity has a Green's function form of:

$$
g(r)=\frac{i}{8 \pi^{2}} \int_{-\infty}^{\infty} \frac{\partial k_{x} \partial k_{y}}{k_{z}} e^{i\left(k_{x} x+k_{y} y+k_{z} z\right)}
$$

related to the Green's Function of the electric field by

$$
G^{\leftrightarrow}=\left[I^{\leftrightarrow}+\frac{\vec{\nabla} \cdot \vec{\nabla}}{k_{1}^{2}}\right] g(r)
$$

Where the symbols are tensors and $k_{1}=\frac{\sqrt{\epsilon \mu}}{c}$.

Power and Final Results

Recalling the fundamental electrodynamic result that:

$$
\langle P\rangle=\int \frac{1}{2} \operatorname{Re}(\vec{J} \cdot \vec{E}) d V
$$

The output power of a quantum emitter in free space, making use of the Green's Function approach presented earlier, can be determinedto be ${ }^{9}$ :

$$
\langle P\rangle=\frac{n \mu \omega^{4}}{3 c^{3}}|\xi|^{2}
$$

Where $\mathrm{n}$ is the material index, $\mu$ the magnetic permeability and $\alpha$ the dipole moment of the photonic emitter.Note that this is a correction to the standard Larmor formula of $\langle P\rangle=\frac{n \omega^{4}}{3 c^{3}}|\xi|^{2}$ previously only observed following arguments from quantum electrodynamics. Taking this result and making use of the semi-classical result based on Fermi's golden rule that:

$$
\Gamma=\frac{P}{\hbar \omega}
$$

Where $\Gamma$ is the photonic density of states. If we were to go back through the procedures presented in this section with the amendment that

$$
G^{\leftrightarrow}=G_{\text {emitted }}^{\leftrightarrow}+G_{\text {reflected }}^{\leftrightarrow}
$$

We would come to the final conclusion that in the presence of a material slab the emitted power, inversely proportional to lifetime, is given by:

$$
\frac{\Gamma}{\Gamma_{o}}=\frac{\tau_{o}}{\tau}=1+\frac{3}{2} \operatorname{Re}\left[\int_{0}^{\infty} \frac{r_{p}(s) S^{3} e^{2 i s_{z} K_{0} * d}}{s_{z}} d s\right]
$$

Where $s^{2}=\frac{k_{x}^{2}+k_{y}^{2}}{K_{0}^{2}} s_{z}^{2}=\frac{k_{z}^{2}}{K_{0}^{2}}, \mathrm{~d}$ is the thickness of the slab, the reflection coefficient of the incident $p$-polarized electromagnetic wave and $\tau$ the lifetime.

9. In this derivation it was assumed that the dipole emitter is pointing up out of the material slab. 


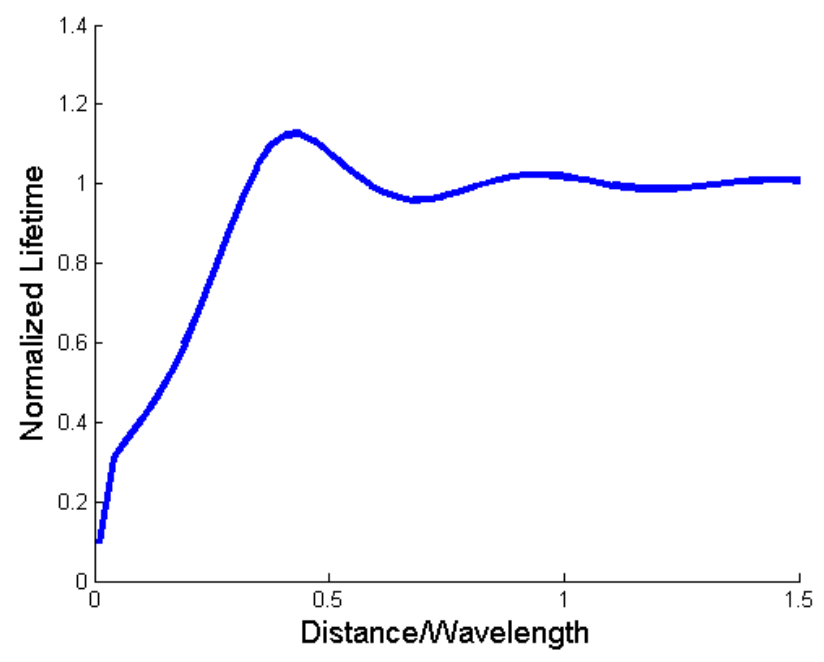

Fig. 7. Sample Quantum Emitter Lifetime Above a Metallic Slab

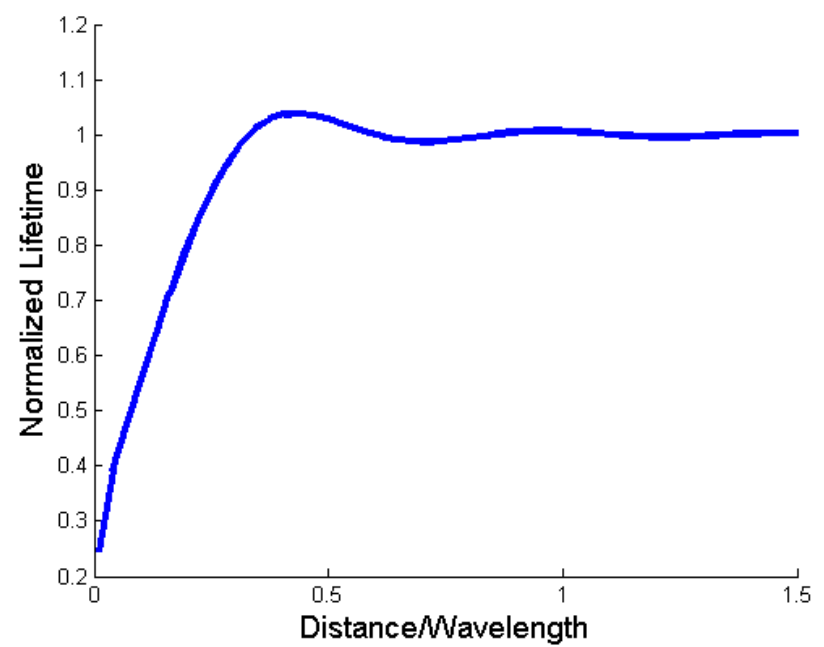

Fig. 9. Sample Quantum Emitter Lifetime Above a Dielectric Slab

\section{CONCLUSIONS}

\section{Lifetime near a Homogeneous Slabs}

Having completed our tour of the theoretical principles involved in calculating the lifetime of a quantum emitter in the presence of any type of material, we can now turn to the final results.

For materials with negative dielectric constants, as the fluorophore descends closer and closer to the interface

more power begins to couple to the plasmonic mode offering some degree of control over the lifetime of the fluorophore. However, as can be seen in the power spectrum plot, the dominant contributions to the enhancement of the photonic density of states are coming from coupling to lossy non radiative modes, a phenomenon known as quenching ${ }^{10}$. As this emitted power may not be extracted from the material for detection the situation is less than ideal.

Materials with strictly positive dielectric constants, as initially predicted, also fail to provide the needed photonic density augmentation to provide a suitable solution to engineering the lifetime of fluorophores. As can be seen from the power spectrum graph of a model dielectric (seen above), the decrease in photonic lifetime in the dielectric case is due to the resonant Fabry-Perot modes

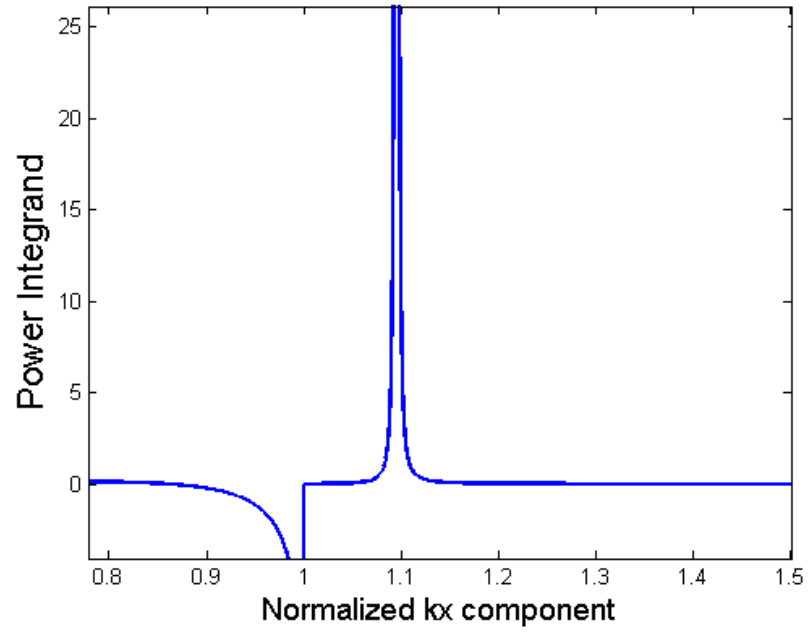

Fig. 8. Sample Quantum Emitter Power Spectrum Above a Metallic Slab

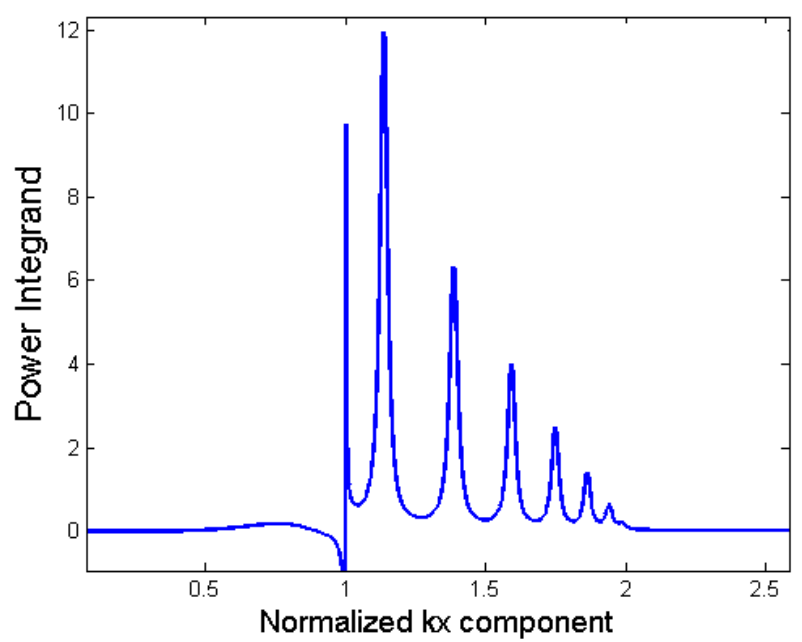

Fig.10. Sample Quantum Emitter Power Spectrum Above a Dielectric Slab

of the slab producing a increase in the photonic density of states via the Purcell effect. As previously discussed in the introduction of this report, this is an unsatisfactory solution in several regards.

\section{Lifetime near Metamaterial Slabs ${ }^{11}$}

Contrary to the plots produced by a homogeneous slab, the modeled behaviour of a quantum emitter above a metamaterial provides significant lifetime reduction with the dominant portion of the power coming from the coupling into surface plasmon modes. With this conformation of our intial inquiry we are ready to produce first order designs of hyperbolic metamaterials for sensing applications.

\section{Designing Metamaterials for Fluorophore Sensing}

Creating the best initial design for HMMs to enhance fluorescence based detection beings with choosing the proper materials. Drawing from the theoretical investigation of the underlying physics of hyperbolic metamaterials, we

10. This can be determined graphically by noting that only one fairly narrow peak exists, yet the lifetime drops quite quickly.

11. The difference between type one and two begin which of the dielectric tensor components is negative. In type 1 this is parallel component; type 2 the perpdicular. 


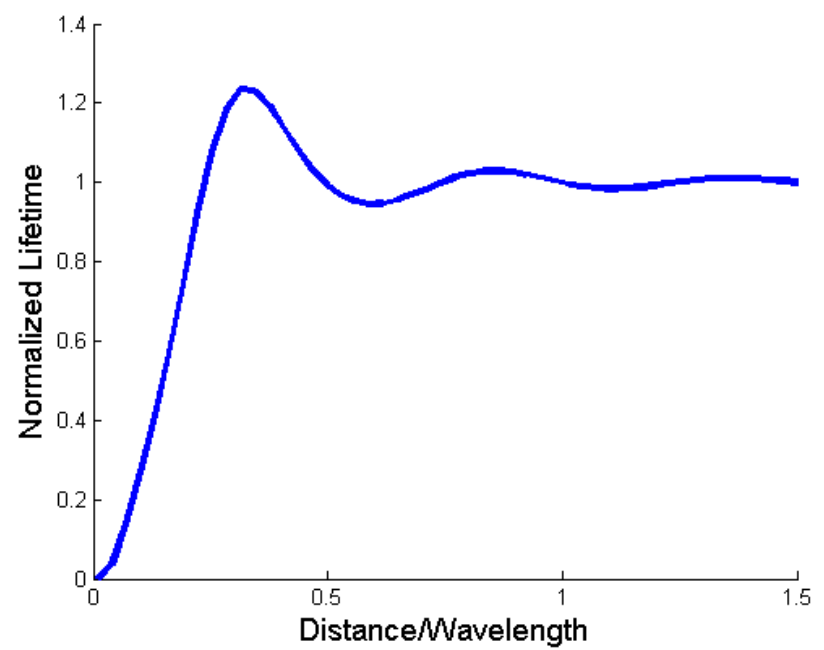

Fig.11. Sample Quantum Emitter Lifetime Above a Slab of Type 1 HMM

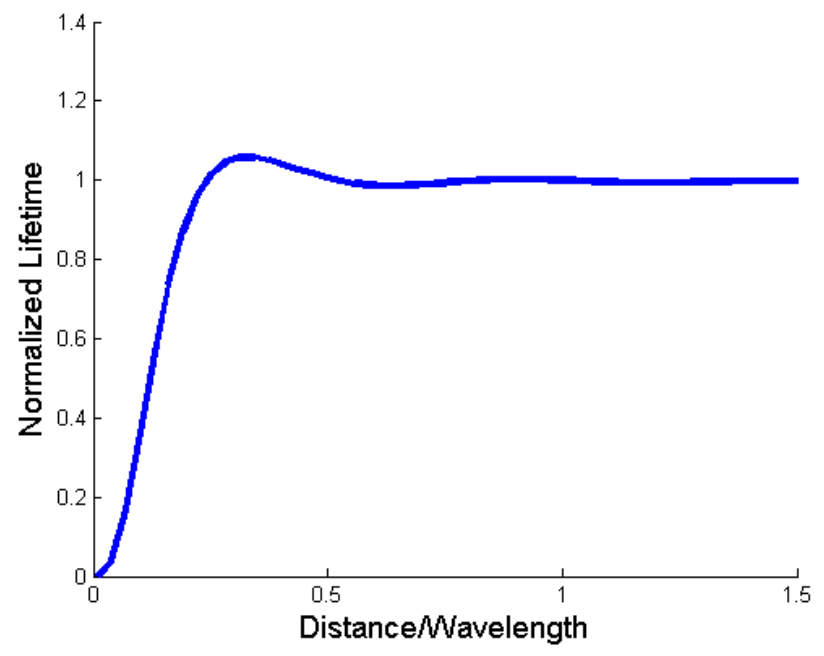

Fig. 13. Sample Quantum Emitter Lifetime Above a Slab of Type 2 HMM

can deduce that in part this amounts to choosing the metal with the smallest imaginary dielectric component, and a complementary dielectric to create reasonable fill factors (14) in the ranges of interest. However, also of concern is the fact that these designs are meant to eventually to provide a basis for creating physical devices, and that as such only materials and procedures which could be accessed by the average high end fabrication facility should be proposed. With these considerations in mind, a careful search dictated by material parameters leads us to focus on Silver, due to it having the smallest imaginary dielectric component as well as favourable properties for fabrication, and $\mathrm{TiO} 2$ for the reasons mentioned above ${ }^{12}$.

The fill factor for these materials can then be calculated at the wavelengths of interest ${ }^{13}$ by setting the value of the dielectric parallel to the interface equal to negative one, in order to provide the best possible coupling as predicted by Maxwell's equations. Leading to the following results:

Taking these results and performing a similar procedure to the work of Joseph Lakowicz [11], leads to us to the conclusion that it is reasonable to assume that HMM designs will offer at least an order of magnitude increase above and beyond the resolution of state of the art silver fluorescence based sensors. Considering the ease

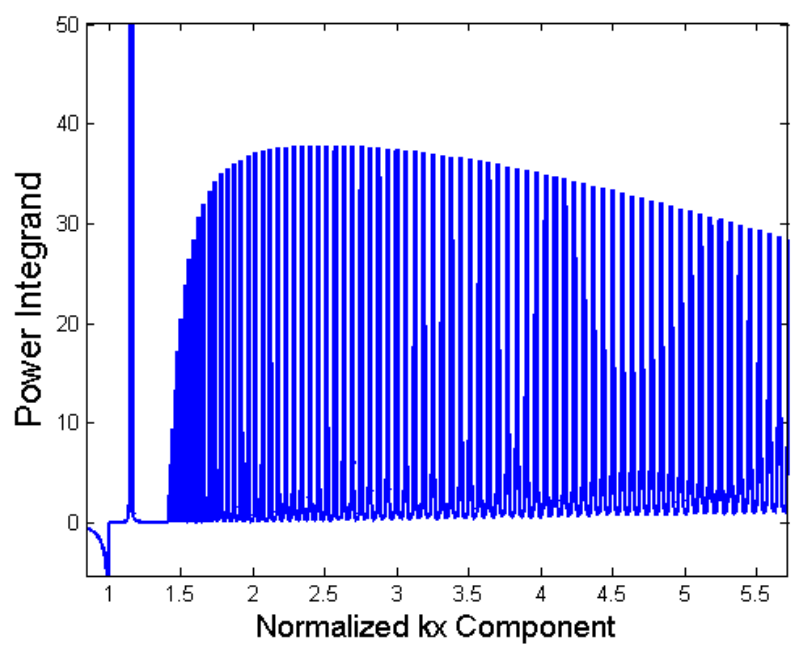

Fig.12. Sample Quantum Emitter Power Spectrum Above a Slab of Type 1 HMM

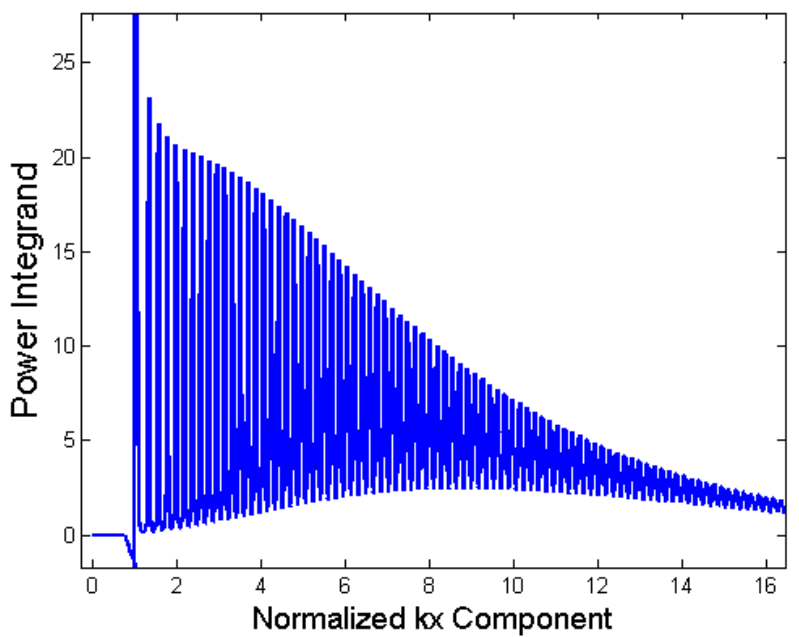

Fig.14. Sample Quantum Emitter Power Spectrum Above a Slab of Type 2 HMM

with which HMM systems can be designed, commerical application is probable and possibly already in progress.

\section{CONCLUSION}

In this article I have presented the basis for understanding how the lifetime of a quantum emitter can be manipulated through the use of hyperbolic metamaterials. Although the details of how a reduced lifetime corresponds to enchanced resolution is left to other authors [12], the basic idea of a flash begin easier to detect than a constant low level source should be reason enough to believe that this is indeed the case. If you have any questions about the article, or would like more information on any of the topics presented, please feel free to email address listed at the beginning of the article.

12. Silver may be deposited down to approximately $11 \mathrm{~nm}$ by sputtering and in thinner films by atomic layer deposition[10]. Ti02 can be deposited over silver ether by chemical vapour deposition or by once again using atomic layer depotion.

13. Six hundred and eight hundred nanometer wavelengths were chosen due to the large number of commonly used fluorophores in these regions, such as QDOT 800 and AlexaFluor 555 (source: Invtrogen). 


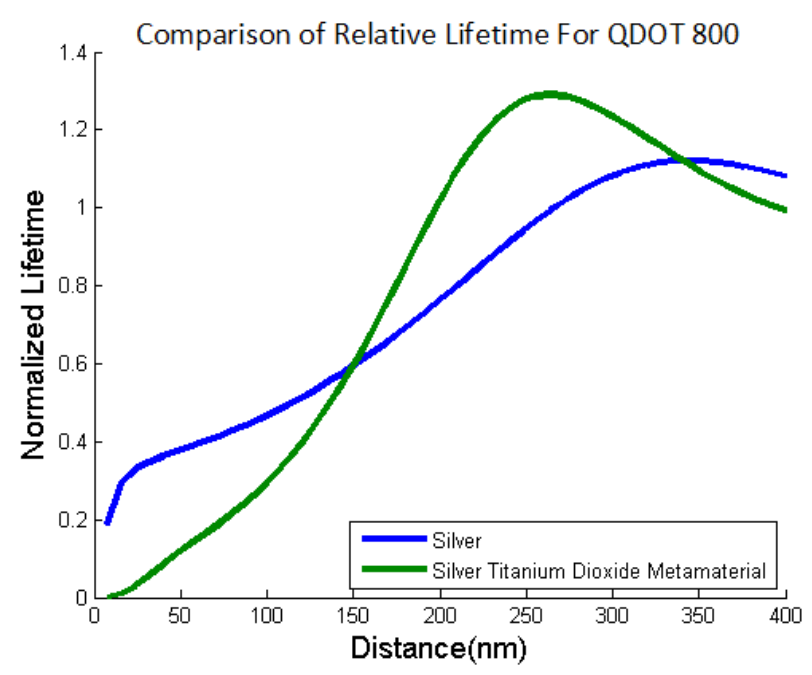

Fig. 15. Comparison of Lifetime Between HMM Design and Current Plasmonic Enhanced Slab at 800nm

\begin{tabular}{|l|l|}
\hline Material & Reflectance Average [\%] \\
\hline Silver $(800 \mathrm{~nm})$ & 86.9 \\
\hline Silver $(600 \mathrm{~nm})$ & 73.6 \\
\hline HMM $(800 \mathrm{~nm})$ & 19.8 \\
\hline HMM $(600 \mathrm{~nm})$ & 19.8 \\
\hline
\end{tabular}

Table 1. Comparison of Reflectance between Metamaterial Design and Current Plasmonic Enhanced Slab

\section{ACKNOWLEDGMENTS}

The work presented in this article was completed as part of a Capstone Design Project in the department of Electrical and Computer Engineering. The author would like to acknowledge Professors Zubin Jacob and Chris Backhouse for their time and guidance throughout the project; and Ward Newman for many insightful discussions.

\section{Reference}

[1] J. M. Gerard and B. Gayral. Strong Purcell effect for InAs quantum boxes in three-dimensional solidstate microcavities. Journal of Lightwave Technology, 17(11):2089-2095, 1999.

[2] S. Noda, M. Fujita, and T. Asano. Spontaneous-emission control by photonic crystals and nanocavities. Nature Photonics, 1(8):449-458, August 2007.

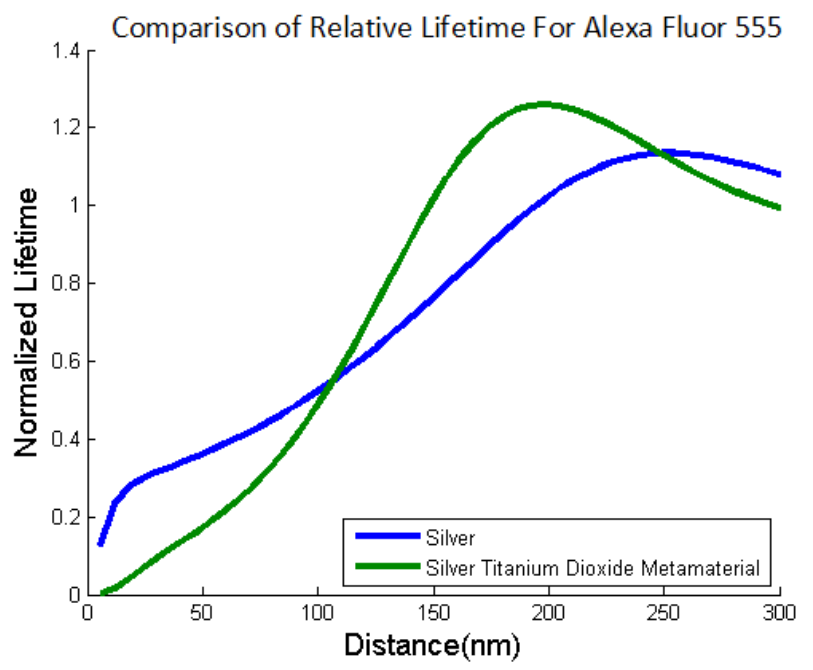

Fig.16. Comparison of Lifetime Between HMM Design and Current Plasmonic Enhanced Slab at 600nm

[3] Z. Jacob, I. Smolyaninov, and E. Narimanov. Broadband Purcell effect: Radiative decay engineering with metamaterials. ArXiv, 0910.3981, October 2009.

[4] L. E. Ballentine. Quantum Mechanics: A Modern Development. World Scientific, Hackensack, NJ, 1998.

[5] J. Gerard, B. Sermage, B. Gayral, B. Legrand, E. Costard, and V. Thierry-Mieg. Enhanced Spontaneous Emission by Quantum Boxes in a Monolithic Optical Microcavity. Physical Review Letters, 81(5):1110-1113, August 1998.

[6] Z. Jacob, J. Y. Kim, G. V. Naik, A. Boltasseva, E. E. Narimanov, and V. M. Shalaev. Engineering photonic density of states using metamaterials. Applied Physics B, 100(1):215-218, June 2010.

[7] Z. Jacob, L. V. Alekseyev, and E. Narimanov. Semiclassical theory of the hyperlens. Journal of the Optical Society of America. A, Optics, image science, and vision, 24(10):A52-9, October 2007.

[8] J. D. Jackson. Classical Electrodynamics. Wiley, 3rd edition, 2007.

[9] Z. Jacob, L. V. Alekseyev, and E. Narimanov. Optical Hyperlens: Far-field imaging beyond the diffraction limit. Optics express, 14(18):8247-56, September 2006.

[10] R. Abermann, R. Kramer, and J. Maser. Structure and internal stress in ultra-thin silver films deposited on MgF2 and $\mathrm{SiO}$ substrates. Thin Solid Films, 52(2):215-229, July 1978.

[11] J. R. Lakowicz. Radiative decay engineering 3. Surface plasmoncoupled directional emission. Analytical Biochemistry, 324(2):153169, January 2004.

[12] J. R. Lakowicz. Radiative decay engineering: biophysical and biomedical applications. Analytical biochemistry, 298(1):1-24, November 2001. 


\section{GENDER-SPECIFIC TREATMENT FOR FEMALE YOUNG OFFENDERS}

Eureka

Volume 3, Number 1 (2012)

Lily Le

Psychology Intern, 2010 - 2011, Turningpoint Program, Alberta Hospital Edmonton

The rate of female youth being charged with serious violent crimes in Canada has more than doubled since 1986, rising from 60 per 100, 000 to 132 per 100, 000 by 2005 (Kong \& AuCoin, 2008). These numbers are much lower in comparison to male young offenders, but the increasing number of female youth entering the juvenile justice system calls for increased attention. Because the juvenile justice system has consisted of mostly males, the system is geared towards males and the specific needs of female young offenders have been overlooked.

Researchers have argued that although both sexes may commit the same violent crimes, such as murder and assault, they are gender-specific in their development and are therefore gender-specific in their pathways to offending as well (Belknap \& Holsinger, 1998). Nonetheless, male delinquency is seen as a behavioral issue that is dealt with through rehabilitation programs that focuses on behavior modification, whereas female delinquency is pathologized and seen as a psychiatric problem (Hartwig \& Myers, 2003). Thus, many young female offenders do not receive the gender-specific programs that they need. Moreover, the treatment literature on female young offenders is scarce in comparison to male young offenders.

Although there has been heightened interest in the idea of gender-specific treatment groups for incarcerated female youth, few programs have been implemented in Canada. Many of the groups for female young offenders in the United States concentrate on specific issues such as substance abuse, not a wide range of topics that this population are affected by and/or focused on preventing delinquent behavior in non-offending girls. Thus, research on the specific needs of female young offenders is required to inform treatment that targets the unique needs of this booming population. Informing a gender-specific programming begins with an increased understanding of how female young offenders differ from male young offenders in essential areas such as their trauma exposure, psychopathology, potential pathways to delinquency, and gender constructs.

\section{Trauma}

Female young offenders have reported experiencing a significant amount of trauma in their lives. Ariga et al's (2007) study on trauma exposure indicated that $76.5 \%$ of the delinquent female adolescents had experienced a traumatic experience, with sexual abuse being the most frequently reported (54.7\%). High reports of sexual abuse among young female offenders have been indicated in many other studies on this population. Siegal and Williams (2003) compared delinquency in girls who were sexually abused as a child to a non-abused group. The results indicated that a larger proportion of arrests were made for female delinquents who had a history of child sexual abuse in comparison to the match group in every category of crime examined (i.e. violent offenses, property offenses). Female delinquents who had a history of sexual abuse were 2.1 times more likely to be arrested than the comparison group. When the rate of offending in both groups was compared in adulthood, females with a history of child sexual abuse were twice as likely to be arrested as adults and the number of arrests was two times greater for a violent offense than when compared with the non-abused group. Although there are young male offenders that report being sexually abused as children, the number of female young offenders with this history is much more significant (Gover, 2004). For example, Alemagno, Shaffer-King \& Hammel's (2006) found that the female young offender participants reported unwanted sexual contact 15 times more than the male young offender participants. Female young offenders have also reported a greater incidence of physical and emotional abuse, along with physical neglect in comparison to male offenders (McCabe, Lansing, Garland and Hough, 2002). Many female young offenders experience violence within their own homes. In one study, families of female young offenders demonstrated high rates of domestic violence and physical abuse (Dixon, Howie, and Starling, 2004). Another study found that female young offenders, in comparison to their male counterparts, reported more physical violence in the home, less family support, and a fear of staying in the home (Alemagno, Shaffer-King, Hammel, 2006).

Trauma is not limited to interpersonal violence, but also includes witnessing violence, being confronted by bad news, and childhood maltreatment. Dixon et al (2005) found that $70 \%$ of the participants in their study reported witnessing a violent crime and $66 \%$ reported being confronted with traumatic news and in another study, $32.8 \%$ of the female young offenders reported being confronted with traumatic news (Ariga et al., 2007). These studies showed that the young female offenders often experienced multiple traumatic experiences.

Hence, the literature on trauma and offending show that females in trouble with the law have experienced victimization at much higher rates than the general population. Young female offenders typically experience more trauma than young male offenders, and are also more likely to be arrested than young male offenders, suggesting that their traumatic history may play a role in their delinquent behavior.

\section{Psychopathology}

Traumatic experiences such as experiencing and witnessing violence often leave devastating psychological repercussions, and since many offenders have experienced trauma, this may increase their risk of having psychological disorders. In comparison to non-offending adolescents, Dixon, Howie \& Starling (2004) found that youth in trouble 
with the law experience more psychological symptoms. Female young offender participants reported significantly more types of traumatic experiences than non-offenders, such as higher levels of personal victimization and exposure to violence. They found that female juvenile offenders report significantly more psychopathology than nonoffenders, with conduct disorder (CD) (91\%), substance abuse/dependence (85\%), alcohol abuse/dependence (56\%), depression (55\%) and post-traumatic stress disorder (PTSD) (37\%) being the most common disorders. Nearly half of the participants in this study also reported attempted suicide. All but one of the females had at least one psychiatric diagnosis and $78 \%$ met the diagnostic criteria for three or more diagnosis.

Specifically, sexual abuse among females is associated with higher rates of psychopathology, especially depression. Gover (2004) examined depression in adolescents who were incarcerated and had a history of child sexual abuse. Both males and females who have been sexually abused exhibited significantly higher levels of depression in comparison to boys and girls who have not been sexually abused. Female survivors of sexual abuse were more likely to experience depression and had the highest levels of depression when compared male survivors. The lowest levels of depression were found in boys who were not sexually abused.

PTSD has also been examined more readily in female juvenile offenders with a history of sexual abuse. Because sexual abuse occurs in much higher rates in females than males, many females with a history of sexual abuse experience PTSD in comparison to males (Ariga et al., 2007). McCabe et al. (2002) found that female delinquents have higher rates of parent reported and self reported psychological symptoms as well as DSM disorders when compared with male delinquents.

Dixon, Howie, Starling and Franzcp (2005) found that among the female young offenders in their study, the largest precipitator of PTSD was sexual abuse (70\%), despite other more frequently reported traumas such as witnessing a violent crime and being confronted with traumatic news. Females who experienced PTSD were more likely to report comorbid diagnosis such as depression, eating disorders, anxiety disorders, psychosis, substance/alcohol abuse and attempted suicide as opposed to males who are more likely to display comorbid attention-deficit/hyperactivity disorder (ADHD) disorder and CD.

Substance and alcohol abuse/dependence are also frequent diagnoses within the young offender population. There is a strong link between substance abuse and criminal activity among both male and female young offenders (Chassin, 2008). In one study, $85 \%$ of female young offenders reported having substance abuse/dependence, and $56 \%$ had alcohol abuse/dependence. Many of these participants reported that they started using drugs to cope with the flashbacks and nightmares associated with their PTSD (Dixon, Howie \& Starling, 2004).

\section{Potential Pathways to Offending}

There have been some ideas as to why the rate of female delinquency has increased over the years. One potential pathway to delinquency may be the due to the victimization of prior abuse and violence that occurred in female's lives as has been discussed earlier in this paper. Being victimized in childhood is a stronger predictor of offending for females than males (Cauffman, 2008).

Feiring, Miller-Johnson and Cleland (2007) found that stigmatization, such as self-blame, abuse-specific shame, and internalizing symptoms, such as PTSD and depression in sexual abuse survivors were related to anger and delinquent behaviors.

Schaffner (2002) suggested that sexual and physical violence against girls has provoked many girls to act out violently themselves. Her study examined the relationship between young female offenders and the violence experienced and witnessed in childhood. Over half of the participants in this study reported witnessing physical, sexual and emotional abuse of others. Many of them had witnessed the abuse of others on numerous occasions. However, very few defined these events as acts of violence or abuse, although a relationship was found between witnessing violence and later offending. The increased violent acts among young female offenders can be attributed to the normalization of witnessing others act out aggressively, often within their hostile home environment. Essentially, it may have become engrained in the females to act out violently in response to anger, as that has become a routine reaction which they have observed throughout their lives.

The increased violent crime by young female offenders could also be their way of fighting back in response to their victimization (Wolfe \& Tucker, 1998). Coping mechanisms that are seen at higher rates in females, like self-harm, provide a sense of control, however, acting out violently towards others may be an alternative method to regain some power. Morton and Leslie (2005) found that female young offenders who were victimized felt the need to regain the power and control that was taken away from them through their abuse experiences. The researchers interviewed clinicians who led therapeutic groups for female young offenders, many of which had a history of sexual abuse. The clinicians expressed that the girls' engagement in delinquent behaviors could be their way of regaining control and power in their lives after feeling powerless in their sexual abuse. The girls' they worked with attempted to gain power and control by putting their needs first, only doing something for others when it would benefit them, and by acting aggressively and manipulatively. As the clinicians pointed out, their methods of gaining power and control would ironically result in incarceration, where power and control were taken from them once again.

Prior victimization among young female offenders may not only be related to an increase in violent offending, but non-violent offending as well. In the United States, many female delinquents are charged with status offenses, (crimes that pertain to specific group of people, in this case, minors) such as running away, many of them fleeing from an abusive environment. Often, females who run away end up living on the streets, where they engage in theft, prostitution, and drug-related activities to survive (Wormer, 2010). Coinciding with idea that female violent offending 
could be used as a way to regain power, these previous non-violent activities may serve the same purpose. In one study, female sex workers with a history of sexual abuse reported that by exchanging sex for money, they felt a sense of control over their bodies (Campbell, Ahrens, Sefl, Clark, 2003).

Females often use drugs and alcohol as a coping mechanism for their trauma and psychopathology and therefore may have to continue to engage in high risk behaviors to fuel their substance abuse. Substance abuse has been shown to play a large role in the increase of female offending (Byrne \& Howells, 2002).

Unfortunately, these methods of survival could put females at greater risk for victimization. For example, a female prostitute with a substance abuse problem may increase her chances of being exposed to more traumas, such as sexual violence. As indicated earlier, many female young offenders who experience trauma often experience psychopathology (or mental health issues) such as PTSD and depression. Unfortunately, the unhealthy coping mechanisms commonly adopted by this group include substance abuse and acting out aggressively to themselves and/or others. This pattern of repeated victimization, psychopathology, and unhealthy coping strategies create a cycle of crime that is difficult to break. Thus, when compared with their male counterparts, female young offenders' pathways to offending and coping mechanisms are different and call for increased attention to the gender constructs that make up these differences.

\section{Gender Constructs and its Influence on Delinquent Behavior}

There is a desperate need for gender-specific programming for at risk youth because males and females develop differently and are taught to act in certain ways according to their gender. They also seem to be affected differently by similar histories. Sex is biologically determined, whereas gender is socially constructed by society. How males and females are gendered in society can help explain the gender specific trauma responses, psychopathology and potential pathways to female offending. In female young offenders who have experienced abuse by males, we can examine society's depiction of masculinity and femininity.

As children, males are often taught to be in control and aggressive and are therefore more prone to act out physically, whereas girls are taught to be submissive and polite and are therefore more likely to internalize their anger. Thus, it is not surprising that when it comes to the need to regain a sense of power, males typically exhibit physical and sexual violence and females are typically depressed and acting out through self-harm or suicide (Covington, 2003). These ideas of the dominant male and passive female are introduced to children as soon as they enter the world. Rough play among boys is tolerated and normal, but is usually not acceptable for girls. Specific toys teach children what type of play is expected of them; boys are usually involved in competitive and aggressive play with cars and actions figures, whereas girls are being prepared for their expected roles of nurturing mother and wife, tending to dolls and using Easy-Bake ovens. A large majority of fairy tales teach boys that they are to be brave and powerful, winning over princesses by slaying evil creatures, whereas girls are taught that happily-ever-afters are achieved when they, the passive in-need-of-rescuing females, are saved by the mighty Prince Charming. Aggression in males is often encouraged and condoned, allowing a culture where physical and sexual violence against females is normal. Females are often taught to be passive and are therefore often the victims of male's violent behavior, especially in sexual violence as indicated earlier.

These ideas that males are to be dominant and powerful, whereas females are to be their subordinates continue to be taught and reinforced in adolescence and adulthood. We are bombarded with these messages in the media; just watch a mainstream rap video and more often than not, there are males surrounded by money, cars and scantily clad women. Studies have illustrated that watching television has an impact on how we view gender roles (Lecroy and Daley, 2001). The ideal nuclear family still consists of the working man who is financially dependent, and the housewife who tends for the children. We live in a "rape-prone" culture, a culture characterized by high rates of rape. Our culture promotes sexual aggression in males and female objectification is common. Examples of this include mainstream pornography which has become increasingly degrading and violent against women, yet is a multi-billion dollar business: $\$ 10$ billion in the U.S. and $\$ 57$ billion worldwide annually (Dunlap, n.d.). In a Canadian survey, $87 \%$ of women reported experiencing sexual harassment ("Measuring Violence Against Women, 2006). Sexual assault occurs in 1/3 females worldwide (The World Bank, 2001). Intimate partner abuse and domestic violence (survivors being mainly females) rank at the top of the list in the number of calls to the police. These ideas not only make it inviting and normal for males to victimize females, but it can convince some females that being victimized by males is a normal part of their gender. Some females, especially those who are victimized repeatedly, do not see abuse to them as wrong, but accept that this is their place in society.

Moreover, young females' sense of identity becomes defined by society's messages of femaleness, coupled with how they are constantly subordinate to their male counterparts. The quest to determining one's self-identity is often already difficult for young females, not to mention female young offenders who attempt to do this while incarcerated. For delinquent females, this sense of identity is often related to being a source of pleasure for males, an identity that would increase risky sexual behaviors and criminal activity. Morton and Leslie's (2005) study illustrates that cultured norms of the passive and receptive sexual female are evident in their sense of self. The definition of sexual activity was unclear to many of the young female offenders in the study, most of whom were sexual abuse survivors. However, the idea that women are sexual objects was evident. Many of these girls reported that their ultimate goal was to be with a partner who loved them. Unfortunately, many of their male partners were abusive and encouraged them to participate in delinquent acts. 
Another study found that female young offenders had difficulty defining themselves as females or individuals, instead basing their sense of self on others, usually their male partners. Moreover, they had poor self-esteem, poor female role models, and a poor view of womanhood. More female participants reported exchanging sex for money and drugs, as well as having a sexually-transmitted infection (STI) in the past six months when compared to the male participants. More than half of the male and female participants reported engaging in sexual intercourse before the age of 13 , a risk factor itself for offending. $62.1 \%$ reported engaging in sexual intercourse without a condom (Alemagno, Schaffer-King and Hammel, 2006).

The ways in which females display their aggression is also gendered. Physical aggression is more typical in boys, whereas relational aggression has been observed more in girls (Crick \& Rose, 2000). Relational aggression can be defined as a form of aggression in which harm is caused to another by threatening or damaging their relationships. Because it goes against conventional gender norms for females to act out violently, they often use relational aggression to hurt others, usually other females. This form of aggression has also been observed to get more sophisticated and complex as children mature into adolescence, including both direct and indirect methods of harming relationships (Crick, Casas, and Nelson, 2002). Although physical aggression amongst females is not as common as male physical aggression, the rate in violent offending amongst female adolescents has ignited, and thus, both physical and relational aggression are deserving of discussion with this population.

Gender constructs shape who girls are and who they will become. Gender roles define girls' responsibilities and rightful place in society. Gender stereotypes constrict girls to how they should think and behave. Hence, genderspecific treatment is needed for young female offenders to explore, critique, re-establish, or even develop their roles and identities as empowered females who are capable of change and success.

\section{Case Illustrations of Gender-specific Programs}

There have been few gender-specific programs that have been implemented for young female offenders in a correctional or treatment facility. In the United States, more steps have been taken in this direction of gender specific programs, especially after the 1992 Reauthorization of Juvenile Justice and Delinquency Prevention Act (JJDP Act) which included the component of gender specific services. ("The Juvenile Justice and Delinquency Prevention Act of 1974," n.d.) Zahn et al. found sixty-two programs aimed at delinquent girls and girls in the juvenile justice system in the United States. Out of these programs, nine were targeted towards young female offenders and had at least one evaluation conducted of the program. Although some of these gender-specific programs are prevention programs and/or conducted in places other than the correctional setting, they revolve around and discuss similar models and themes that may be applicable to young female offenders. The programs listed below focus on gender specific issues for at risk youth in a variety of settings, and have been found to produce significant changes in the lives of many female adolescents.

One popular program, Girls' Circle has trained more than 500 organizations and has been implemented in schools, clinics, agencies, homes and group homes. Girls' Circle is a strengths-based group utilizing a model that combines relational theory, resiliency practices, and skills training with the goal of enhancing courage and growth in the group. Gender specific topics include being a girl, friendship, body image, diversity and identity ("How It Works," 2011). In 2007, Roa, Irvine, and Cervantez conducted a study on the effectiveness of Girls' Circle and found that the group improved girls' well-being. Short-term effects include feeling good about their body and expressing verbal affection to others, whereas long-term effects included a decrease in self-harm and alcohol use and an increase in self-efficacy. However, girls' held in juvenile detention, residential facility or other secure facilities did not display as much as an improvement when compared to other girls' who were not in these settings. Gains in short-term skills and self-efficacy of girls in a secure setting were lower than girls who were not. As suggested by the researchers, girls in detention may have specific needs in regards to these short-term skills and self-efficacy and further steps need to be taken to understand the experiences of this specific population. Overall, Girls' Circle has shown to be beneficial to many young females.

In North Carolina, the Holistic Enrichment for At-Risk Teens (HEART) program has been designed to reduce recidivism and substance abuse relapse among young female offenders. The program uses gender-specific perspectives, such as feminist and relational theories along with the Multilevel Risk Model which takes into account the multiple levels of risks (biological, psychological, developmental, familial/communal and societal) that play a role in female delinquency. The program uses the BioPsycho-Social-Spiritual (BPSS) model of addiction which states that many factors, such as biological, psychological, social, spiritual, and developmental, may play a role in substance abuse/dependence. Therapy and groups cover topics such as female socialization, gangs, relational aggression, and sisterhood (Welch, Roberts-Lewis, and Parker, 2009). Girls in the HEART program improved more in their use of social support, perceived support of friend, and peer acceptance when compared with the non-treatment group. Girls in the HEART program also experienced improved self esteem, social development family relationships, and educational status in relation to the comparison group. Also, school engagement and grades improved in the treatment group (Kirk and Griffith, 2004, as cited in Zahn, 2009).

A state-wide day treatment program program in Florida called PACE (Practical Academic Cultural Education) Center for Girls, Inc. is a prevention program provided for girls who may be involved in risky activities or crime. The program recognizes the relationship between prior victimization and crime and therefore provides a gender responsive approach to education, counseling and career planning. The purpose of the program is to prevent female delinquency and risky behaviors by strengthening educational and vocational 
areas in the girls' lives ("Our Goals," 2010). A 2009/2010 evaluation of the program indicated that for girls who were transitioning in 2007-2008, 93\% were not adjudicated nor had adjudication withheld while they were enrolled in the program, 94\% were not adjudicated nor had adjudication withheld within 6 months from the day program, and 91\% were not adjudicated nor had adjudication withheld within 1 year from the day program. Academic goals, such as having at least $25 \%$ of the girls mainstream back into a public school and having at least $75 \%$ of the girls increase their academic functioning (i.e. GPA, specific subject assessments, earning credits and completing courses) were all exceeded with $64 \%$ and $95 \%$ of girls meeting both goals, respectively. Altogether, 21,000 girls have been served across the 17 PACE centers and programs in Florida. PACE's focus on girls' strengths and protective factors have illustrated that participants who complete the program are able to make more positive choices, such as concentrating on educational and vocational goals, which prevent delinquency and high risk behaviors.

Lastly, the Go Grrrls Program is an after school prevention program for middle-school girls that aims to foster healthy gender role identification and healthy psychosocial development in today's society. The program focuses on gender specific issues such as body image, self-esteem, friendship, sex, success, and independence. Lecroy (2004) conducted an evaluation of the program. Participants were divided in to a treatment group who attended the program, and a control group who did not attend the program. Before and after each intervention, participants filled out self-questionnaires with 8 different measures such as body image, self efficacy, and assertiveness. The evaluation found that for variables such self-efficacy and self-liking and competence, girls in the program displayed a significant positive change in comparison to the control group. Moreover, a significant between group effect was found, with the treatment group reporting a greater increase in body image, assertiveness, attractiveness, and self liking and competence than the control group.

With little gender specific programs for incarcerated female delinquents, there is a scarcity of literature on the evaluations of these programs. There is research to show that gender specific treatment does work, especially when the programs cover multiple aspects of the girls' lives (Cauffman, 2008). Although gender-specific treatment has indicated that although gender-specific programming does have positive effects in areas such as education, relationships, self-esteem, self-efficacy and other socialpsychological outcomes like body image, there is little evidence to illustrate that this type of programming reduces recidivism, despite improving girls' quality of life. This may suggest that these programs work while girls remain in the program (Zahn et al., 2009). Covington (2000) stressed the importance of creating a safe space through site and staff selection, program development, content and material that depicts the truths of the lives of girls and women in our society.

There have been implications from experts in the field on what key elements are required to facilitate successful gender-responsive programming for females. It has been suggested that because female delinquents often use anger as a mode of survival, programs should begin with topics such as self-esteem building, empathy training and maintaining healthy boundaries to build strength within them and therefore be less likely to resort to anger to gain power (Morton \& Leslie, 2005). Garcia and Lane (2009) found that a common desire amongst young female offenders in their girls' group was the desire to have a voice and play an active role in decision-making regarding their care. These girls longed for staff to show them that they cared. These considerations, along with the successful elements of case illustrations mentioned should be taken into account and form a basis for future gender specific programs for young female offenders.

\section{The Turningpoint Program}

The Turningpoint program at Alberta Hospital is an 18-bed facility that strives to provide the best possible inpatient assessment, treatment, and rehabilitation for youth in trouble with the law. This mission is accomplished by utilizing best practices in treatment and sparking new research. Although there has been an increase in the number of female young offenders admitted for treatment and assessments over the years, there has been no treatment group that addresses the needs of girls specifically. As supported by the literature, the young female offenders who stay at the Turningpoint program are violent offenders who often have experienced trauma and have been diagnosed with a number of psychopathologies. Many come from backgrounds where they have witnessed and experienced violence. Many have had a history of repeated victimization. Most commonly, these girls share the similar experience of being a young woman in trouble with the law, and thus share a number of gendered experiences. With the majority of the young offenders on the unit being male, these girls do not have a space where they can come together to discuss gender specific issues. Thus, forming a gender specific group for the female young offenders will provide the opportunity for these girls to have a safe environment where their experiences can be acknowledged, where they can learn about their gender specific issues, and where they can be empowered to make positive life changes.

\section{The Turningpoint Girls' Group}

Similar to the case illustrations discussed, the Turningpoint Girls' Group will use strengths-based, empowerment approach and focus on a variety of topics from a female perspective. Like the PACE Center for Girls, Inc., the group will emphasize the relationship between childhood abuses and delinquency ("Our Goals," 2010). Thus, the group is rooted in the feminist pathways perspective, which suggests that delinquent behaviors may derive from a number of areas that exist within our patriarchal society, such as learned gender roles, structural oppression, female's victimization by males, and female's responses to male power. This theory posits that patriarchy plays a large role in delinquency and therefore, both boys' and girls' potential pathways to delinquency may be gender specific (Belknap \& Holsinger, 2006). The theory is not limited to just gender, but other intersections such as 
race and class.

Belknap \& Holsinger (2006) determine how feminist pathways theory contributes to better understanding the gendered risks associated with delinquency. For measures of abuse, girls reported significantly more abuse than boys for almost every variable. For measures of family, girls had the worst home experiences, reporting significantly more abandonment than boys, and reporting that someone else had helped raise them aside from their parents more than boys. In terms of mental health, girls reported significantly higher likelihood of mental health problems. For measures of school experiences, girls were significantly more likely than boys to drop out of school or quit. Consistent with the literature presented on gender specific issues relating to female delinquents, the feminist pathways theory provides the most support for determining not only girls' risks, but boys' risks as well. Feminist pathways theory has illustrated the importance of understanding how trauma is a risk factor for delinquency, and thus needs to be incorporated into other theories that do not address this issue when working with delinquents. The majority of gender-specific programs examined in this study targets similar risk and protective factors, and a few others target specific risk factors such as substance abuse. However, very few target abuse and neglect, family and peer relationships, mental health and gang involvement although these risk factors are extremely influential in the pathway to delinquency. All in all, the findings of girls' experiences are most consistent with feminist pathways theory and thus life events of delinquent females, such as traumatic experiences, must be considered for effective gender specific programming.

In terms of counseling paradigms, an empowerment/ strengths-based approach will be taken in the Turningpoint Girls' Group. Cognitive-behavioral therapy has served as the dominant paradigm in corrections. This approach focuses on changing offenders' thinking patterns, offenders' pathology, and preventing recidivism. The strengths-based approach, on the other hand, focuses on multiple interactive levels of influence, the person as a whole, and the offenders' sense of motivation to change and well being. This approach is rooted in the belief of human potential (Wormer, 2010).

The group will be available for all female-identified patients on the unit, both treatment patients (those who are admitted to the treatment program) and remand patients (those who are not admitted to the treatment program, but awaiting assessment). The group will be psychoeducational, presenting information and ideas through education and activities regarding a variety of genderspecific issues in which the girls can relate with. Similar to previous groups that have been conducted with this population, the girls' group will cover topics such as selfesteem, self-identity, anger, healthy relationships (familial, peer, and romantic), sexuality, risk and protective factors, gender specific trauma, and girls in relation to the world. Education and discussion of these issues will hopefully promote growth, healing and empowerment for these girls. Discussion of the issues will be encouraged and done so in a safe and supportive environment. The group will always take place in a quiet room off the unit to instill consistency, safety and comfort. Weekly sessions of 1-1.5 hours will take place over a course of 8-12 sessions. Pre and post self-evaluations measuring concepts such as self-esteem, knowledge, and overall satisfaction of the group will be handed out to all the patients. Homework will be given and collected on a weekly basis.

The group will be facilitated by the author, along with another female co-facilitator. These facilitators will be aware of the girls' traumatic experiences, offense(s), current mental health, and needs. Facilitators will work with the feminist pathways theory in mind, and using an empowerment approach to foster a sense of confidence within the group. It is key that facilitators develop good rapport with the girls, be sensitive to their history and current mental state, and work to foster an environment of trust.

The author is hopeful that gender-specific treatment for the young female offenders of the Turningpoint program will be helpful in promoting positive change in the girls' lives. Perhaps with the implementation of this program over time, gender specific programming for this population will be of greater interest in Canada and the specific needs of delinquent females will be less likely to be overlooked.

\section{References}

Alemangno, S., Shaffer-King, E., \& Hammel, R.(2006). Juveniles in detention: How do girls differ from boys? Journal of Correctional Health Care, 12(1), 45-53.

Ariga, M., Uehara, T., Takeuchi, K., Ishige, Y., Nakano, R., \& Mikuni, M. (2007). Trauma exposure and posttraumatic stress disorder in delinquent female adolescents. The Journal of Child Psychology and Psychiatry, 49(1), 79-87.

Belknap, J., \& Holsinger, K. (1998). An overview of delinquent girls: How theory and practice failed and the need for innovative changes. In R. T. Zaplin (Ed.), Female Offenders: Critical Perspectives and Effective Interventions, Gaithersburg, MD: Aspen Publishers.

Belknap, J., \& Holsinger, K. (2006). The gendered nature of risk factors for delinquency. Feminist Criminology, 1(1), 48-71.

Byrne, M., \& Howells, K. (2002). The psychological needs of women prisoners: Implications for rehabilitation and management. Psychiatry, Psychology and Law, 9(1), 34-43.

Campbell, R., Ahrens, C., Sefl, T., \& Clark, M. (2003). The relationship between adult assault and prostitution: An exploratory analysis. Violence and Victims, 18(3), 299-317.

Cauffman, E. (2008). Understanding the female offender. The Future of Children, 18(2), 119-134.

Chassin, L. (2008). Juvenile justice and substance use. The Future of Children, 18(2), 165-183.

Covington, S. (2000). Creating gender-responsive programs: The next step for women's services. Corrections Today, 63(1), 85-87.

Covington, S. (2003). A woman's journey home: Challenges for female offenders. In J. Travis \& M. Waul (Eds.), Prisoners once removed: The impact of incarceration and reentry on children, families, and communities. Washington, DC: Urban Institute Press.

Crick, R., \& Rose, A. (2000). Toward a gender-balanced approach to the study of socio emotional development: A look at relational aggression. In Miller, P., \& Scholnick, E. Toward a Feminist Developmental Psychology. New York, NY: Routledge.

Crick, R., Casas, F., \& Nelson, A. (2002). Toward a more comprehensive understanding of peer maltreatment: Studies of relational victimization. Current Directions in Psychological Science, 11(3), 98-101.

Dixon, A., Howie, P., \& Starling, J. (2004). Psychopathology in female juvenile offenders. Journal of Child Psychology and Psychiatry, 45(6), 1150-1158. 
Dixon, A., Howie, P., Starling, J., \& Franzcp, J. (2005). Trauma exposure, posttraumatic stress, and psychiatric comorbidity in female juvenile offenders. American Academy of Child and Adolescent Psychiatry, 44(8), 798-806.

Dunlap, B. (n.d.) Porn, meth and violence: Making some connections. Crisis Connection. Retrieved from http://www.crisisconnectioninc. org/pdf/Porn.pdf

Feiring, C., Miller-Johnson, S., \& Cleland, C. (2007). Potential pathways from stigmatization and internalizing symptoms to delinquency in sexually abused youth. Child Maltreatment, 12(3), 220-232.

Garcia, C., \& Lane, J. (2009) What a girl wants, what a girl needs: Findings from a gender-specific focus group study. Crime \& Delinquency, published online April 3, 2009. Retrieved from http:// cad.sagepub.com/content/early/2009/04/03/001112

Gover, A. (2004). Childhood sexual abuse, gender, and depression among incarcerated youth. Internation Journal of Offender Therapy and Comparative Criminology, 48(6), 683-696.

Hartwig, H., \& Myers, J. (2003). A different approach: Applying a wellness paradigm to adolescent female delinquents and offenders. Journal of Mental Health Counselling, 25(1), 57-75.

How It Works. (2011). Girls Circle. Retrieved from http://www.girlscircle. com/ how it works.aspx

Kirk, R., \& Griffith, D. (2004). End-of-year report to the Department of Juvenile Justice and Delinquency Prevention for state fiscal year 2004: Evaluation summary of assessment information for residents of the HEART and BEST programs at the Samarkand Youth Development Center. Chapel Hill: University of North Carolina, Chapel Hill, Jordan Institute for Families, School of Social Work. Cited by Zahn, M., \& Day, J. (2009). Determining what works for girls in the juvenile justice system: A summary of evaluation evidence. Crime \& Delinquency, 55(2), 266-293.

Kong, R., \& AuCoin, K. (2008). Female offenders in Canada. Juristat: Canadian Centre for Justice Statistics, Statistics Canada, Catalogue 85-112-XIE, 28(1).

Lecroy, C. (2004) Experimental evaluation of "Go Grrrls" preventive intervention for early adolescent girls. The Journal of Primary Prevention, 25(4), 457-473.

Marx, M. PACE outcome measure evaluation report (July 1, 2009 - June 30, 2010). PACE. Retrieved from http://www.pacecenter.org/images/ stories/PACE_Outcome_Measures_Report_0\%20-10.pdf
McCabe, K., Lansing, A., Garland, A., \& Hough, R. (2002). Gender differences in psychopathology, functional impairment, and familial risk factors among adjudicated delinquents. American Academy of Child and Adolescent Psychiatry, 41(7), 860-867.

Measuring Violence Against Women: Statistical Trends. (2006). Statistics Canada, Catalogue no. 85-570-XIE.

Morton, G., \& Lesley, L. (2005). The adolescent female delinquent: A feminist developmental analysis. Journal of Feminist Family Therapy, 17(1), 17-50.

Our Goals. (2010). PACE. Retrieved from http://www.pacecenter.org/

Roa, J., Irvine, A., \& Cervantez, K. (2007). Girls Circle national research project. Girls Circle. Retrieved from http://www.girlscircle.com/docs/ Final_Report_2007.pdf.

Schaffner, L. (2007). Violence against girls provokes girls' violence: From private injuryto public harm. Violence Against Women, 13(12)12291248.

Siegel, A., \& Williams, M. (2003). The relationship between child sexual abuse and female delinquency and crime: A prospective study. Journal of Research in Crime and Delinquency, 40(1), 71-97.

The Juvenile Justice and Delinquency Prevention Act of 1974: Prior Federal Juvenile Delinquency Activity. (n.d.). Retrieved from Office of Juvenile Justice and Delinquency Prevention, http://www.ojjdp.gov/ compliance/jidpchronology.pdf

The World Bank. (2001). Cited in Worldwide Sexual Assault Statistics. (2005). George Mason University Sexual Assault Services.

Welch, C., Roberts-Lewis, A., \& Parker, S. (2009). Incorporating gender specific approaches for incarcerated female adolescents: Multilevel Risk Model for practice. Journal of Offender Rehabilitation, 48(1), 67-83.

Wolfe, L., \& Tucker, J. (1998). Report of the Summit on Girls and Violence. Washington, DC: Center for Women Policy Studies.

Wormer, Katherine Van. (2010). Working with female offenders. Hoboken, New Jersey: John Wiley \& Songs, Inc.

Zahn, M., \& Day, J. (2009). Determining what works for girls in the juvenile justice system: A summary of evaluation evidence. Crime \& Delinquency, 55(2), 266-293. 

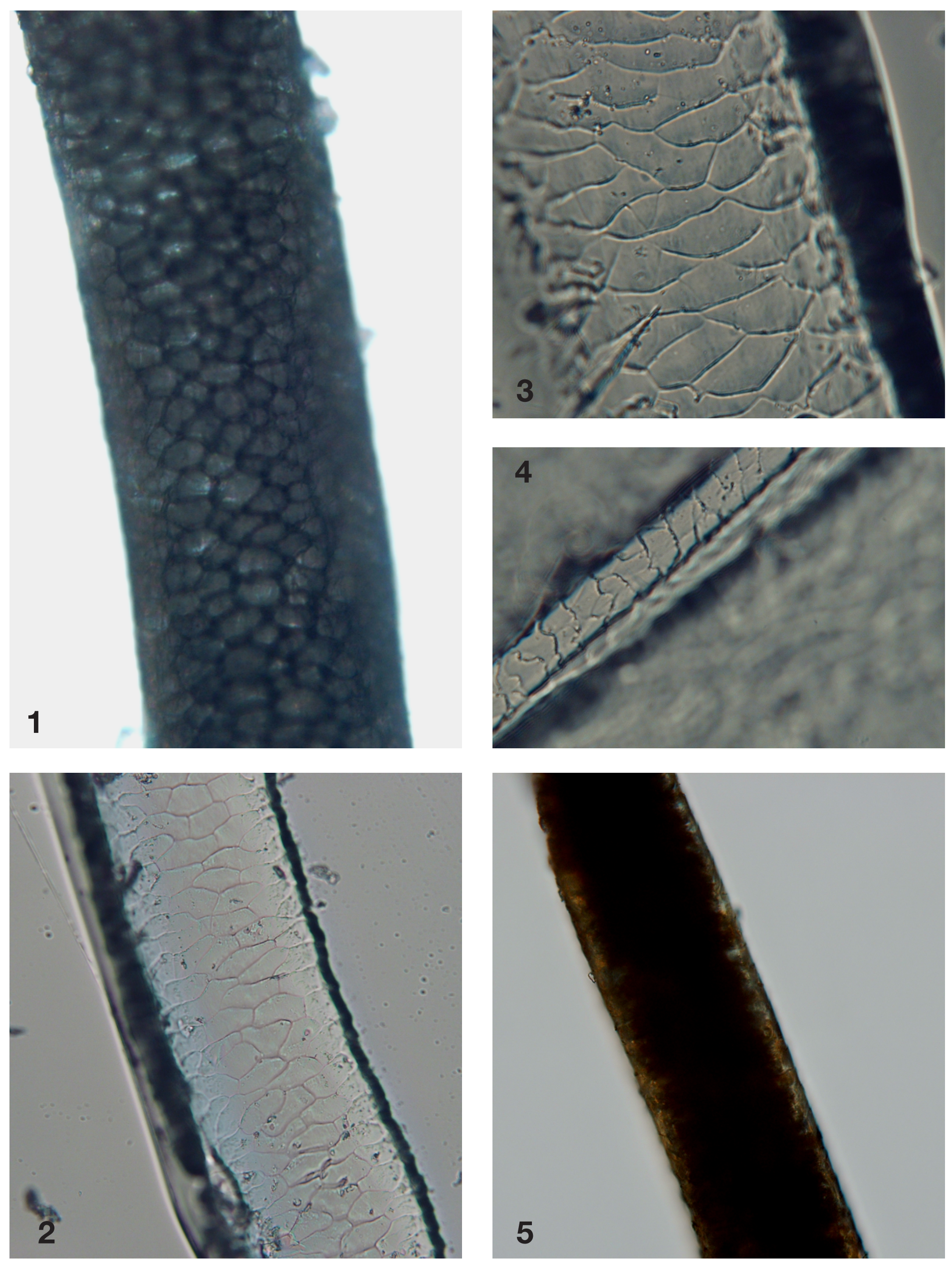

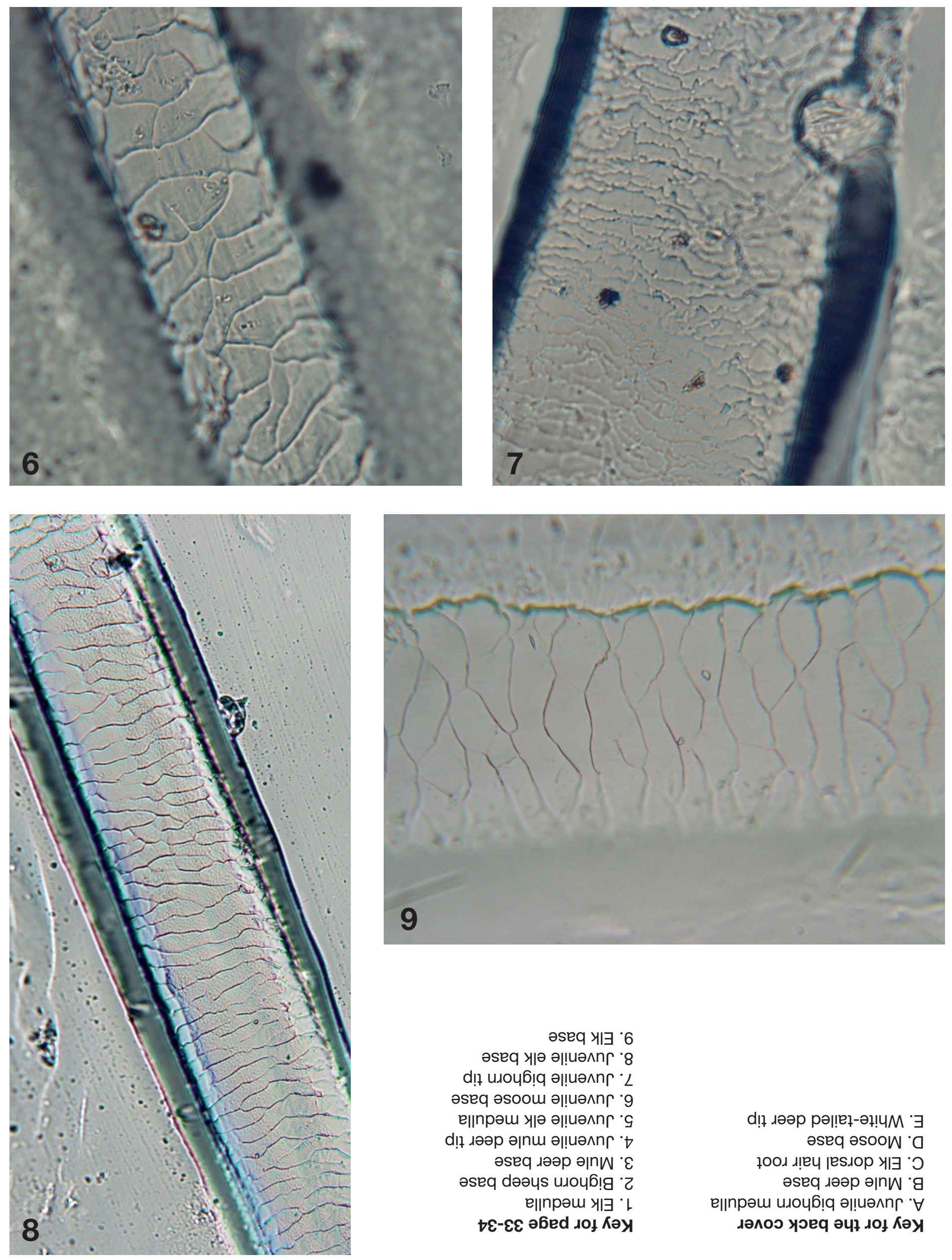

әseq 빜.6

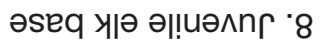

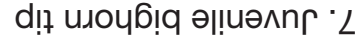
әseq әsoou ə|!

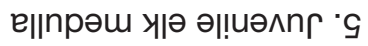

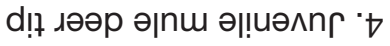
әseq әseq dəəus uхоц6!! ' 2 е॥прәш 빜 '

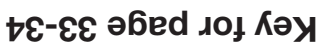

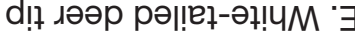
əseq əsooW 'C

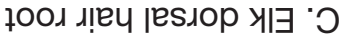
әseq גәәр әाn' 'q

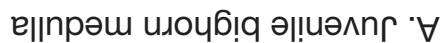

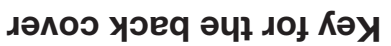



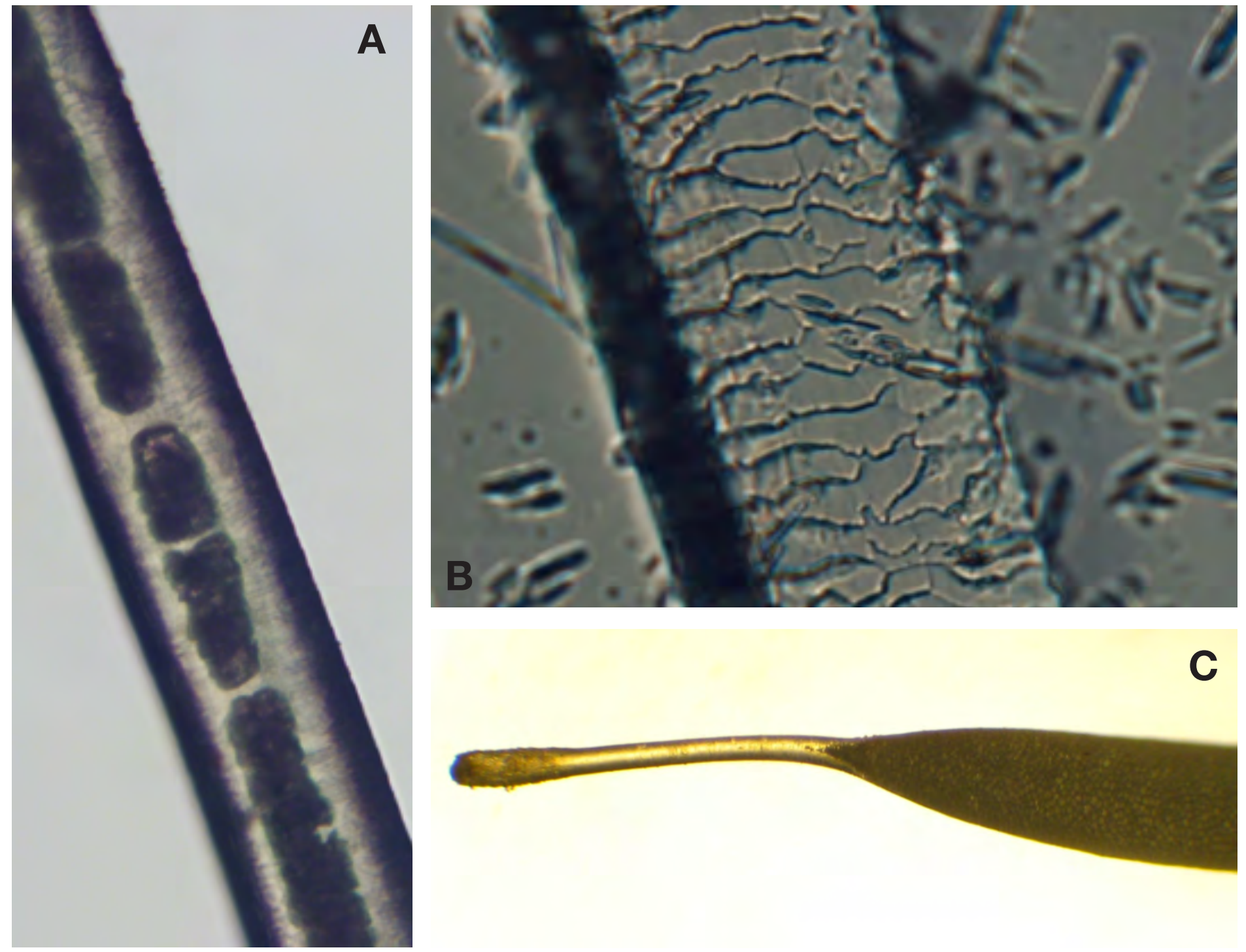

C

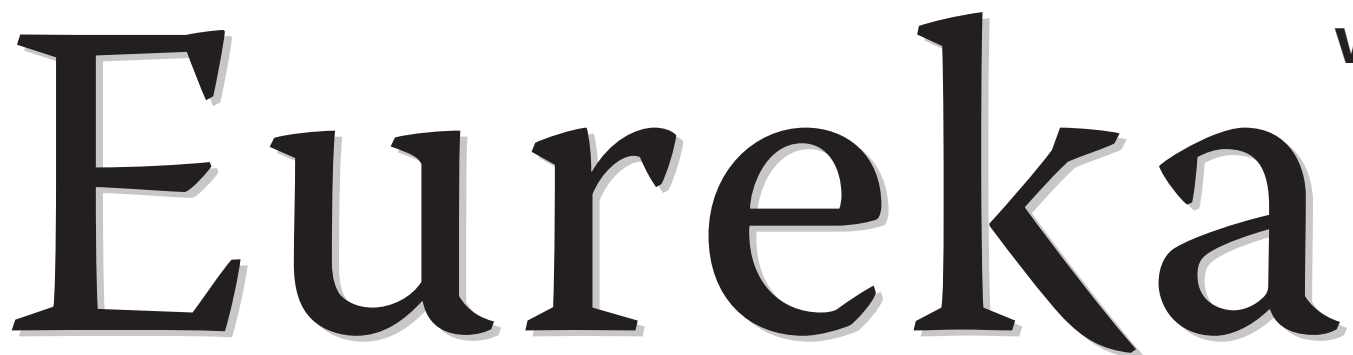

Volume 3, Number 1

February 2012

University of Alberta Science Undergraduate Research Journal

http://www.eurekajournal.com
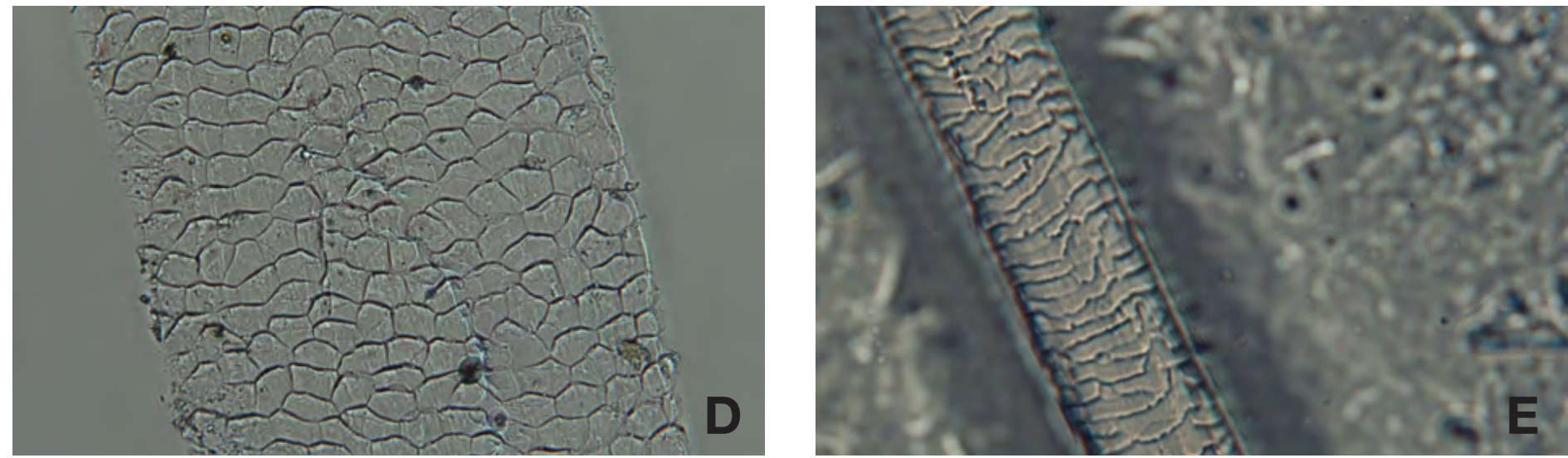